\title{
Helical vortex core dynamics and flame interaction in turbulent premixed swirl combustion: A combined experimental and large eddy simulation investigation
}

Cite as: Phys. Fluids 31, 025108 (2019); https://doi.org/10.1063/1.5065508

Submitted: 10 October 2018 . Accepted: 09 January 2019 . Published Online: 05 February 2019

S. Taamallah, Y. Dagan (D, N. Chakroun, S. J. Shanbhogue (D), K. Vogiatzaki, and A. F. Ghoniem

\section{COLLECTIONS}

EP This paper was selected as an Editor's Pick

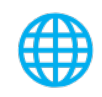

PHYSICS TODAY WHITEPAPERS
ADVANCED LIGHT CURE ADHESIVES

Take a closer look at what these

environmentally friendly adhesive

systems can do
READ NOW

PRESENTED BY 8. MASTERBOND: 


\title{
Helical vortex core dynamics and flame interaction in turbulent premixed swirl combustion: A combined experimental and large eddy simulation investigation
}

\author{
Cite as: Phys. Fluids 31, 025108 (2019); doi: 10.1063/1.5065508 \\ Submitted: 10 October 2018 - Accepted: 9 January 2019 • \\ Published Online: 5 February 2019
}

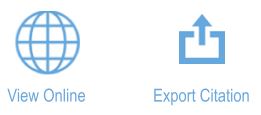

\section{S. Taamallah, ' Y. Dagan, 'D N. Chakroun,' S. J. Shanbhogue, 'D K. Vogiatzaki, ${ }^{2}$ and A. F. Ghoniem}

\author{
AFFILIATIONS \\ ${ }^{1}$ Massachusetts Institute of Technology, 77 Massachusetts Ave., Cambridge, Massachusetts 02139, USA \\ ${ }^{2}$ University of Brighton, Cockcroft Building, Lewes Road, Brighton, East Sussex BN2 4GJ, United Kingdom
}

\begin{abstract}
In this study, a combined experimental and Large Eddy Simulation (LES) investigation is performed to identify the vortical structures, their dynamics, and interaction with a turbulent premixed flame in a swirl-stabilized combustor. Our non-reacting flow experiment shows the existence of large scale precessing motion, commonly observed for such flows. This off-axis precessing dynamics disappears with combustion but only above a critical equivalence ratio at which the flame attaches to the swirler centerbody and vortex breakdown changes from a cone to a bubble type. For compact flames stabilized along the inner shear layer (ISL), no precessing is seen, but large scale vortices along the ISL are observed; these structures interact with the ISL-stabilized flame and contribute to its wrinkling as revealed by laser-induced fluorescence data. After validating the LES results in terms of low order statistics and point temperature measurements in relevant areas of the flow, we show that it can capture the precessing motion in the non-reacting flow and its suppression with combustion. The simulations show that the ISL vortices in the reacting case originate from a vortex core that is formed at the swirler's centerbody. This vortex core has a conical helical shape that interacts-as it winds out-with the ISL and the flame stabilized along it. The simulated helical vortex core (HVC) exists in both reacting and non-reacting flows; in the latter, it is dominated by the offaxis motion, whereas in the reacting case, that motion is damped and only remains the cork-screw type solid body rotation of the HVC.
\end{abstract}

Published under license by AIP Publishing. https://doi.org/1 0.1 063/1.5065508

\section{INTRODUCTION}

Swirling flows are widely encountered. In nature, the most prominent are found in tornadoes ${ }^{1}$ as well as in our blood vessels where such flows enhance oxygen transfer. ${ }^{2}$ In technology, they are present in aircraft wingtip vortices, ${ }^{3}$ some heat exchanger designs, ${ }^{4}$ cyclone separators,$^{5}$ and modern low emission gas turbine engines which are the main focus of this paper. In the combustion system of these engines, fuel and air are premixed for a better control of the burnt gases temperature to maintain acceptably low thermal nitrogen oxides levels. Because premixed flames are not bound to any particular location in the flow by considerations of stoichiometry, ${ }^{6}$ they are subject to blowoff ${ }^{7}$ at low equivalence ratios. Swirling flows were introduced to provide a robust anchoring of these flames ${ }^{8,9}$ and extend the lean blowoff limit.

Considerable efforts have been made toward understanding these flows. ${ }^{9-21}$ Premixed flames in swirl flows stabilize along shear layers around recirculation zones such as the inner or central recirculation zone (IRZ) introduced by a vortex breakdown (VB). Vortex breakdown appears above a critical swirl strength because of the associated large radial and axial pressure gradients that create a stagnation point and a zone of recirculation of hot products adjacent to the incoming reactant jet. Vortex breakdown can also be due to combustion at operating conditions where it would not take 
place for the non-reacting flow [combustion induced vortex breakdown $\left.(\mathrm{CIVB})^{10}\right]$. Multiple types of vortex breakdowns have been observed over the years although at high Reynolds numbers, the "bubble" and the "spiral" types were found to be predominant. ${ }^{18}$ While vortex breakdown helps stabilize a premixed flame, the same mechanism can be also detrimental because of the presence of unsteady vortical structures that interact with the flame and potentially lead to heat release disturbances. Unsteady heat release induced by large vortical structures can couple with the acoustic field and potentially lead to undesired thermoacoustic instabilities. ${ }^{22}$

Two groups of large structures exist in swirling flows:

1. Kelvin-Helmholtz $(\mathrm{KH})$ instability induced structures: 23 in a non-swirling axisymmetric jet flow, $\mathrm{KH}$ instability gives rise to vortex rings. In swirling flows, the combined axial-azimuthal shear layer gives rise to a helical vortex that winds around the shear layer. These instabilities were analyzed, ${ }^{24}$ examined experimentally, ${ }^{25}$ and modeled numerically. ${ }^{26,27}$ In addition to the inner shear layer (ISL) at the interface between the incoming swirling jet and the vortex breakdown zone, an outer shear layer (OSL) often exists in swirling flows in combustion. This OSL, with its own vortical structures, is formed downstream of a sudden expansion dome often present between the burner and the main combustion chamber.

2. Helical instability-induced structures: above a certain swirl strength, the inner swirling core can develop selfsustained oscillations and transitions to a limit cycle regime following a supercritical Hopf bifurcation. ${ }^{28}$ In this oscillatory state, the vortex core is in circular motion around the geometrical centerline in a precessing type motion called a helical vortex core (HVC) ${ }^{29}$ or a precessing vortex core (PVC) that dominates the flow.

The above vortical structures modulate the mixing processes between the fuel, air, and hot combustion products ${ }^{20}$ and thus affect the heat release rate. They also wrinkle and stretch the flame, and depending on the sign of the stretch rate and the Lewis number (Le), the reactivity and the heat release rate can be enhanced (positive stretch with sub-unity Le) or reduced. In turn, heat release and associated thermal expansion and baroclinic vorticity can affect the vortical structures. For instance, the precessing motion of a PVC can be damped and can eventually disappear. ${ }^{19,30}$ Recent linear stability analyses in reacting flows ${ }^{31}$ show that the temperature and density profiles are key in triggering large scale azimuthal oscillatory motion; this has recently been used to explain why non-reacting swirling flows and swirling lifted flame are associated with the existence of a large scale precessing dynamics. ${ }^{29}$ Similarly, when a swirling flame attaches to a centerbody, the precessing motion was shown to be damped.

\section{A. Goal and outline of the current paper}

While the recent effort to study flow and flame dynamics and structures in turbulent combustion remained strong, both experimentally ${ }^{32-36}$ and numerically, ${ }^{37-42}$ very few studies joined the strengths of experimental and numerical tools to gain insight into the vortical structures that we observe in such reacting flows. The goal of this work is to combine experiments and simulations in order to identify the origin and dynamics of flame and flow structures in a model swirl burner.

This paper is organized as follows: we start with a description of the experimental setup, along with the different diagnostic and numerical tools used. Next, a brief but necessary validation for the Large Eddy Simulation (LES) code is performed relying on experimental Particle Image Velocimetry (PIV) data (flow field statistics) and temperatures in some relevant zones of the flow [outer recirculation zone (ORZ) and downstream]. Then, the experimental evidence of large scale flow and flame structures and their dynamics are shown and analyzed. Finally, LES of the same geometry is shown in which we track the origin of the vortical structures observed in the experiment. The simulation gives access to the entire three dimensional field and thus complements the detailed but mainly two-dimensional optical diagnostics used in our investigation. This will allow us to unveil the vortical structures' origin, dynamics, and interaction with the premixed reaction zone.

\section{EXPERIMENTAL AND NUMERICAL SETUPS}

\section{A. Experimental apparatus}

We study a fully premixed swirl-stabilized combustor (Fig. 1) similar to the one used in our recent studies. ${ }^{43,44} \mathrm{~A}$ circular $38 \mathrm{~mm}$ inner diameter stainless steel premixing tube starts with a choke plate, whose purpose is to prevent acoustic fluctuations from affecting the equivalence ratio. The inlet tube contains an axial swirler with a streamlined centerbody. The swirler has eight flat blades with an angle $\alpha_{s w}=45^{\circ}$ that is fixed throughout this study [leading to an estimated swirl number $\left.\mathrm{S} \approx 2 / 3 \tan \left(\alpha_{s w}\right) \approx 0.7\right] \cdot{ }^{20,45}$ The burner inlet tube continues after the swirler up to a sudden expansion with a ratio $D_{\text {out }} / D_{\text {in }}=2$. A $2.5 \mathrm{~mm}$ thick fused silica round tube (uncooled) with twice the inner diameter of the inlet pipe $(76 \mathrm{~mm})$ is used for confinement and optical access. A circular opening of $10 \mathrm{~mm}$ diameter located $10 \mathrm{~mm}$ downstream of the expansion plane allows us to insert a K-type thermocouple and record the ORZ temperature $\left(\mathrm{TC}_{1}\right)$. The thermocouple protrudes by $5 \mathrm{~mm}$ from the wall into the ORZ. A similar type thermocouple $\left(\mathrm{TC}_{2}\right)$ is inserted downstream and located on the centerline. Methane is used as the fuel in this work and was supplied by a Sierra C100M Smart-Trak digital mass flow controller with a flow rate uncertainty of $\pm 1 \%$ of maximum capacity. A Sierra Instruments 780 S Flat-Trak flow meter is used to measure the air flow rate with the same uncertainty. The subsequent measurement error on the equivalence ratio is $\pm 2 \%$.

\section{B. Operating conditions}

The two main cases studied experimentally and numerically in this paper are a non-reacting flow at a Reynolds 


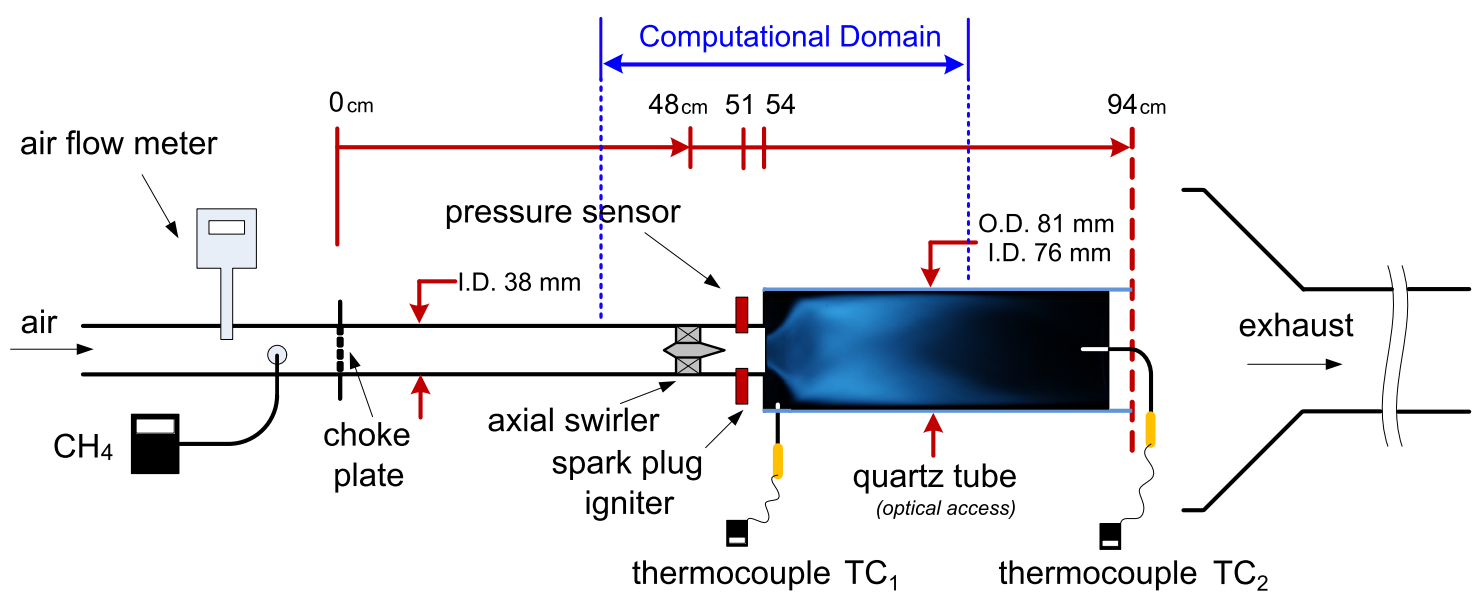

FIG. 1. Schematic of the swirl-stabilized experimental combustion system. The LES computational domain is also shown.

number (Re) of 20000 and a swirl number (S) of 0.7 (45 angle) and a premixed $\mathrm{CH}_{4}$-air reacting case, with the same $\mathrm{Re}$ and $\mathrm{S}$, at an equivalence ratio $\phi=0.60$. These are labeled as the non-reacting and the reacting cases throughout this study. In both cases, the inlet air-fuel mixture temperature is ambient and the pressure atmospheric. At $\phi=0.60$, a specific mode of flame stabilization is observed, which is the inner shear layer (ISL) flame. At these conditions, we estimate that the flame is in the reaction sheet regime (or thickened-wrinkled flamelet) as displayed by the turbulent combustion Borghi-Peters diagram in Fig. 2. Owing to the presence of different zones in the swirling flow with substantially different turbulent intensity, the outer recirculation zone (ORZ) flow and flows along the inner and outer shear layers are placed separately on the diagram. In the Borghi-Peters diagram, the integral length

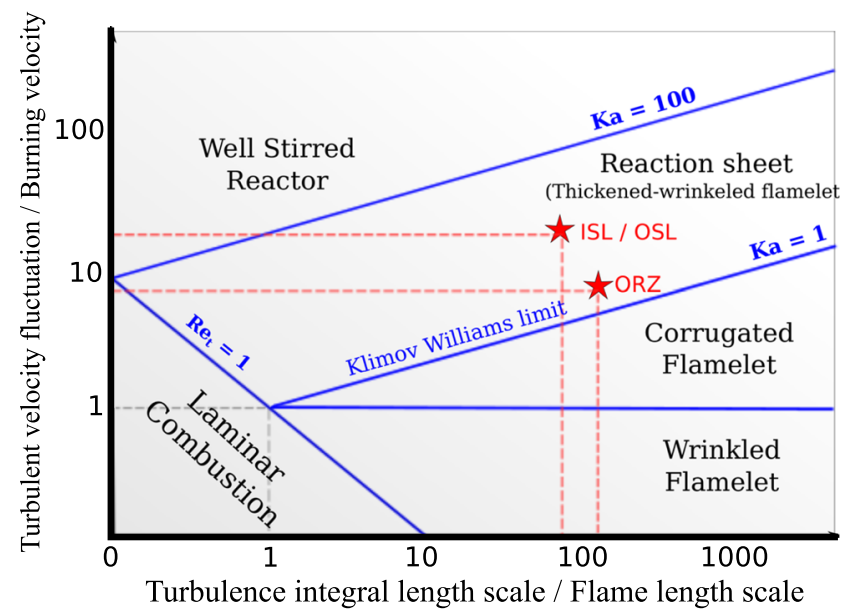

FIG. 2. Borghi-Peters diagram for turbulent combustion regimes. The two red stars correspond to the estimated values for the main reacting flow case studied in this paper (premixed $\mathrm{CH}_{4}$-air combustion, $\phi=0.60$, $\mathrm{Re}=20000, \mathrm{~T}_{\text {inlet }}=300 \mathrm{~K}$, atmospheric pressure); one for the flow conditions in the outer recirculation zone (ORZ) and one for the flow conditions in the inner and outer shear layers (ISL and OSL). scale used for the shear layers is the largest scale of flame wrinkles observed in hydroxyl radicals planar laser induced fluorescence images (OH-PLIF, which will be shown later), whereas the integral length scale for the ORZ was considered equal to the step height.

Other flame stabilization modes (referred to as flame macrostructures) are observed for other equivalence ratios, and these will be described later. Although the focus of this paper will be on the non-reacting and reacting reference cases mentioned above, additional cases at different equivalence ratios or different Reynolds number will be used to support our discussion or LES validation when needed. The operating conditions are summarized in Table I.

\section{Diagnostic tools}

High speed chemiluminescence flame imaging recorded at $1 \mathrm{kHz}$ with an infrared (IR) blocking filter (a 2-mm thick CG-BG-39 Schott glass) was performed using a NAC GX1 high-speed CMOS camera mounted above the combustor. This method provides high temporal resolution, but it suffers from being a line of sight measurement and having a relatively long exposure giving access only to the flame brush.

We relied on OH-PLIF to get the instantaneous flame location and structure in the centerplane with high spatial resolution but without temporal resolution $(10 \mathrm{~Hz})$. PLIF images were acquired by exciting $\mathrm{OH}$ species at $283.56 \mathrm{~nm}$ wavelength using a Spectra Physics LAB 170 pump laser with a Sirah Cobrastretch dye laser, circulating Coumarin 153 dye dissolved in ethanol. Fluorescence from excited species taking place at $308 \mathrm{~nm}$ was optically filtered and intercepted by using a $1280 \times 1024$ pixel LaVision Nanostar CCD camera, with $2 \times 2$ binning. OH-PLIF measurements were performed at $10 \mathrm{~Hz}$ with a gating time of $100 \mathrm{~ns}$. A laser beam analysis system including a beam splitter and an Ophir Photonics SP620U CCD camera was used for beam analysis. The OH-PLIF raw images were corrected for the spatial and temporal non-uniformity of the laser sheet and background noise filtered. 
TABLE I. Operating parameter space.

\begin{tabular}{lccccc}
\hline \hline Cases & $\mathrm{Re}_{\text {inlet }}$ & $\alpha_{\text {sw }}(\mathrm{deg})$ & Fluid & $\phi$ & Other cases \\
\hline Non-reacting & 20000 & 45 & Air & & $\mathrm{Re}=15000-30000$ \\
Reacting & 20000 & 45 & $\mathrm{CH}_{4}$-air & 0.60 & $\phi=0.48-0.65$ \\
\hline \hline
\end{tabular}

We used a two-dimensional, two-component high-speed particle imaging velocimetry (PIV) system to interrogate the reacting flow field. For non-reacting flows, high-speed stereoscopic PIV was performed giving access also to the azimuthal component of the turbulent velocity field in the same twodimensional interrogated plane. Aluminum oxide $\mathrm{Al}_{2} \mathrm{O}_{3}$ seeding particles (of diameter between 0.9 and $2.2 \mu \mathrm{m}$ ) were introduced into the main air flow upstream of the swirler. A light sheet less than $1 \mathrm{~mm}$ thick is produced along the combustor's axis using a $50 \mathrm{~W}$ Nd:YLF laser. A high-speed NAC GX-1 CMOS camera is mounted above the combustor, recording images at $1 \mathrm{kHz}$. Data using two different fields of view were recorded to obtain different resolutions: one with a relatively large field of view $(120 \mathrm{~mm} \times 76 \mathrm{~mm})$ encompassing the entire diameter of the chamber as well as the entire inner recirculation zone $(\mathrm{dx}=\mathrm{dy}=1.4 \mathrm{~mm})$; the other with a narrower field of view $(50 \mathrm{~mm} \times 40 \mathrm{~mm}$ ) focused on the inner and outer shear layers with a higher resolution of $\mathrm{dx}=\mathrm{dy}=0.6 \mathrm{~mm}$. At each operating condition, 538 pairs of images were recorded and repeated three times. A statistical uncertainty analysis on the PIV data was performed by computing the standard error of the mean; the mean velocity is computed for each of the three ensembles at the reference inflow conditions and $\phi=0.60$ at the location of maximum mean axial velocity; we obtain $\mu_{1}=12.27 \mathrm{~m} / \mathrm{s}, \mu_{2}=12.37 \mathrm{~m} / \mathrm{s}$, and $\mu_{3}=12.32 \mathrm{~m} / \mathrm{s} \mathrm{lead}-$ ing to a relative standard error of the mean of $1 \%$ (with $95 \%$ confidence).

In addition to optical diagnostics, temperature measurements were performed. A K-type sheathed thermocouple $\left(\mathrm{TC}_{1}\right)$ was inserted through the quartz tube $10 \mathrm{~mm}$ downstream of the sudden expansion side wall and $5 \mathrm{~mm}$ into the ORZ. $\mathrm{TC}_{1}$ has a sheath diameter of $1 / 16$ in. and a response time around $1 \mathrm{~s}$. ORZ temperature is sampled at $20 \mathrm{~Hz}$. Multiple measurements were made to evaluate an uncertainty of $\pm 5 \mathrm{~K}$. $\mathrm{TC}_{1}$ is used to measure the relatively low temperature of the unburnt reactants in the ORZ before the appearance of a flame in that zone. The temperature measurements in this location can be subject to radiation error from different sources: radiative heating of the probe by the hot chamber walls and by the hot products. Radiation errors in $\mathrm{TC}_{1}$ measurements due to gas radiation were neglected for the following reason: by changing the diluent from $\mathrm{N}_{2}$ (air combustion) to $\mathrm{CO}_{2}$ (oxy-combustion) at the same adiabatic flame temperature, the change in $\mathrm{T}_{\mathrm{ORZ}}$ was within the error margin $(5 \mathrm{~K})$, suggesting limited radiation exchange between the probe and products. The probe measurement was also not corrected for the radiation error from hot walls: when comparing $\mathrm{T}_{\mathrm{ORZ}}$ for different levels of insulation of the chamber (which leads to different wall temperatures), only a $30 \mathrm{~K}$ temperature difference was measured by the probe. This increase was attributed to the combination of lower heat losses at the boundary and higher artificial heating of the probe by the hot wall. The temperatures obtained by the probe are considered as upper bounds for the ORZ gas temperature.

It should be pointed out that in the current work, all the experimental diagnostic tools are two-dimensional and nonsimultaneous. In order to collect additional information for the instantaneous 3D dynamics, we will rely on complementary LES computations.

\section{Computational method}

\section{Governing equations, SGS models, and discretization}

A truncated domain from the previously described experimental setup is modeled. The truncated domain includes the swirler and a part of the combustion chamber $(225 \mathrm{~mm}$ out of $400 \mathrm{~mm}$ of the round quartz tube is modeled); the extent of the numerical domain can be seen in Figs. 1 and 3. The LES code, similar to that described in previous studies by Kewlani et al., ${ }^{46-48}$ was implemented using, as a basis, OpenFOAM ${ }^{\circledR}$ 's C++ libraries which is based on a finite volume method for spatial discretization. An implicit second order temporal scheme is used along with a combination of first and second order schemes for spatial discretization balancing numerical stability and accuracy. Few differences with the previous work ${ }^{46-48}$ relate to the grid, the choice of numerical schemes and the parameter choice for the combustion model.

The partial differential equations (PDEs) governing the flow are the mass, linear momentum, and sensible enthalpy conservation and are closed using the ideal gas law as equation of state. Reactions are taken into account using the reduced mechanism of Jones and Lindstedt ${ }^{49}$ containing 7 species and 5 reactions. The residual subgrid scale stress tensor is closed using Prandtl's one equation model based on the turbulent kinetic energy (TKE) which uses the Boussinesq eddy viscosity postulate. The filtered reaction rate is closed using an Arrhenius rate law similar to a DNS approach which is one of the advantages of the artificially thickened flame combustion (ATF) model used here and will be described in the following paragraphs.

In the ATF model, a thickening factor F multiplies the flame thickness and the effective (sum of molecular and SGS: $\mathrm{D}_{\text {eff }}$ ) diffusivity maintaining the propagation of the flame locally at the laminar burning velocity. The thickened flame can then be resolved on the numerical grid, but an efficiency function $\mathrm{E}$ must be used to recover the flame-flow interactions and heat release. This efficiency function is modeled using the algebraic expression proposed by Colin et al. ${ }^{50}$ 


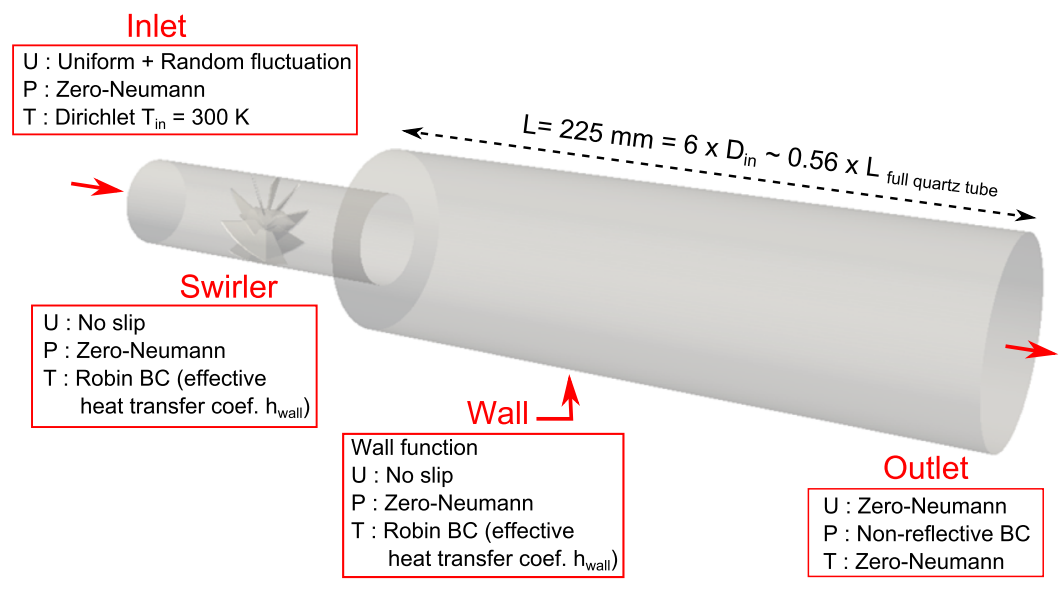

FIG. 3. LES computational domain and boundary conditions.

A dynamic thickening approach is implemented here where $\mathrm{F}$ and $\mathrm{D}_{\text {eff }}$ scalars are used to build associated fields $\left(\mathrm{F}_{\text {loc }}\right.$ and $\left.\mathrm{D}_{\text {eff,loc }}\right)$ that depend on the location relative to the flame. ${ }^{51}$ In the ATF combustion model, the filtered species conservation becomes (with $\widetilde{Y}_{k}$ the filtered mass fraction of species k)

$$
\frac{\partial \bar{\rho} \widetilde{Y}_{k}}{\partial t}+\frac{\partial \bar{\rho} \widetilde{u}_{i} \widetilde{Y}_{k}}{\partial x_{i}}=\frac{\partial}{\partial x_{i}}\left(\bar{\rho} F_{l o c} E D_{e f f, l o c, k} \frac{\partial \widetilde{Y}_{k}}{\partial x_{i}}\right)+\frac{E \overline{\dot{\omega}_{k}}}{F_{l o c}} .
$$
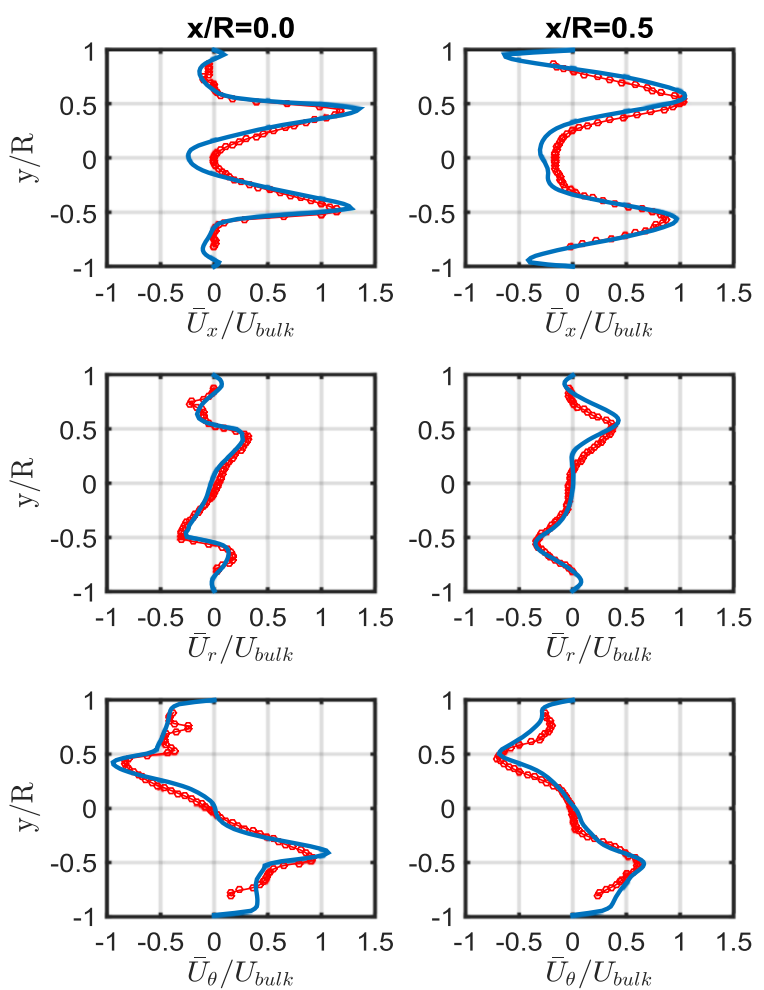
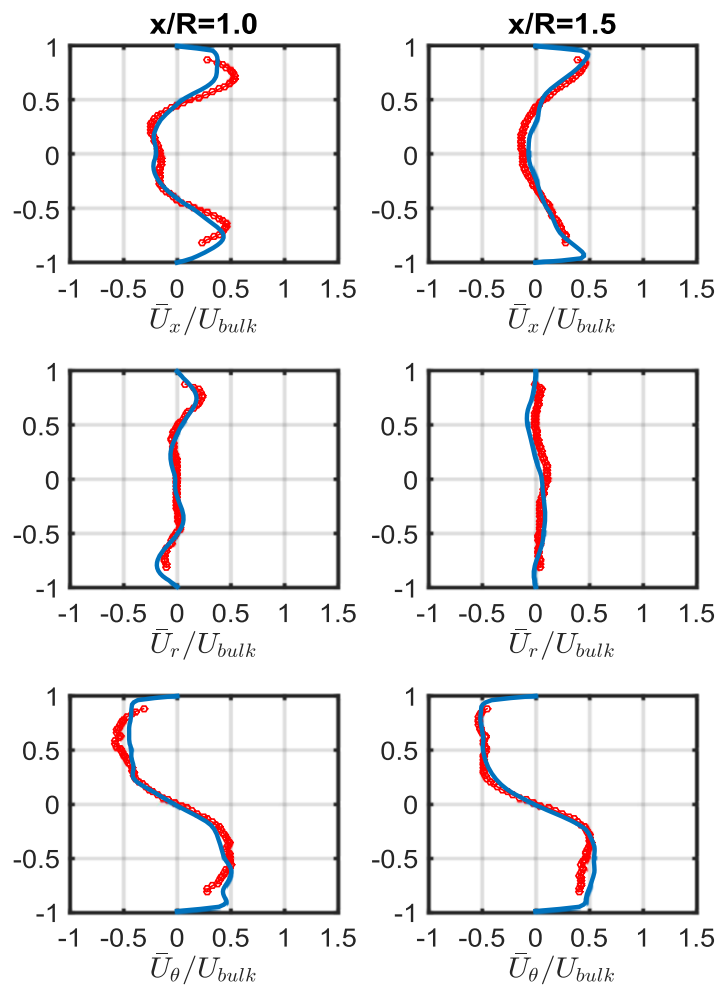

FIG. 4. Non-reacting mean velocity profiles: Experimental (red circle) vs. LES. $R=D_{i n} / 2$ is the combustion chamber radius. 
The terms $\boldsymbol{\mu}$ and Sc are the dynamic viscosity and the Schmidt number, respectively.

The efficiency function is calculated using the local filter width $\Delta$, the unstretched laminar burning velocity $S_{L}^{0}$, the laminar flame thickness $\delta_{\mathrm{L}}^{0}$ and thickened flame thickness $\delta_{\mathrm{L}}^{1}$, and the local SGS velocity fluctuation $u_{\Delta}^{\prime}$ following the expression suggested by Colin et al., ${ }^{50}$

$$
E=\frac{\Xi\left(\delta=\delta_{L}^{0}\right)}{\Xi\left(\delta=\delta_{L}^{1}\right)},
$$

with the non-dimensional wrinkling factor $\Xi$,

$$
\Xi(\delta)=1+\alpha\left(\frac{2 \ln (2)}{3 C_{m s}\left(\operatorname{Re}_{t}^{1 / 2}-1\right)}\right)\left(\frac{u_{\Delta}^{\prime}}{S_{\mathrm{L}}^{0}}\right) \Gamma\left(\frac{u_{\Delta}^{\prime}}{S_{\mathrm{L}}^{0}}, \frac{\Delta}{\delta_{\mathrm{L}}^{0}}\right),
$$

and

$$
\Gamma\left(\frac{u_{\Delta}^{\prime}}{S_{L}^{0}}, \frac{\Delta}{\delta_{L}^{0}}\right)=0.75\left(\frac{\Delta}{\delta_{L}^{0}}\right)^{2 / 3} \exp \left[-1.2\left(\frac{u_{\Delta}^{\prime}}{S_{L}^{0}}\right)^{-0.3}\right]
$$

\section{Computational domain, grid, and boundary conditions}

The computational domain and the main boundary conditions are summarized in Fig. 3. A mixed hexahedral and tetrahedral mesh is constructed where hexahedral cells are used everywhere except around the swirler and its centerbody to better capture all its geometrical details. The grid is refined mostly around the shear layers where large gradients are expected. A larger grid size is gradually employed as we move downstream. A maximum cell size of $\Delta x_{\max }=1.6 \mathrm{~mm}$ is chosen based on the criteria of resolving at least $80 \%$ of the turbulent kinetic energy (the integral length scale chosen to be the expansion step height of $19 \mathrm{~mm}$ ). The refinement where large gradients are expected led to a minimum cell size of $\Delta x_{\text {min }}=0.32 \mathrm{~mm}$. This ultimately led to a grid size of $0.65 \times 10^{6}$ cells with an average cell size $\overline{\Delta x} \approx 1 \mathrm{~mm}$. The average cell size imposed a choice of a thickening factor $F$ used by the combustion model. As the filter size $(\Delta)$ is taken to be the cell size, a thickening factor $\mathrm{F}=3$ was chosen allowing approximately five grid points across the thickened flame to resolve it,

$$
\mathrm{F} \times \frac{\alpha_{\text {th }(800 \mathrm{~K})}}{\mathrm{S}_{\mathrm{L}(\phi=0.6)}^{0}} \approx 5 \times \overline{\Delta x},
$$

where $\alpha_{\text {th }}$ is the thermal diffusivity and $S_{L}^{0}$ is the laminar unstrained flame speed.

For brevity, no grid sensitivity tests are reported in this paper, but such tests can be found in previous work. ${ }^{46,47}$ The thermal boundary condition used at the wall is a Robin type condition that takes into account an effective external heat transfer coefficient $\left(\mathrm{h}_{\text {wall,effective }}\right.$ in $\left.\mathrm{W} \mathrm{m} \mathrm{m}^{-2}\right)$; a value of $\mathrm{h}_{\text {wall,effective }}=50 \mathrm{~W} \mathrm{~m}^{-2}$ is estimated using a thermal resistance
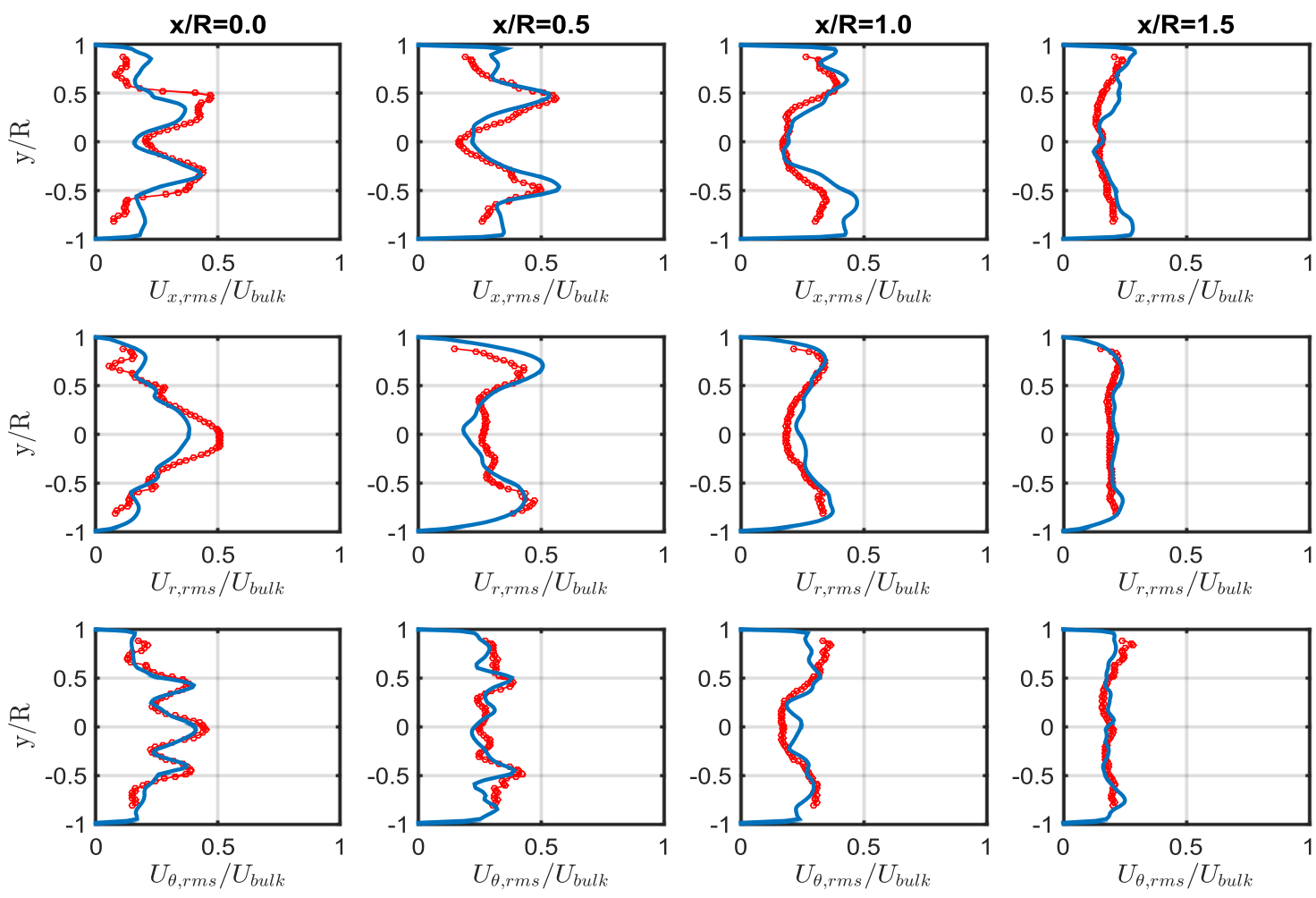

FIG. 5. Non-reacting RMS velocity profiles: Experimental (red circle) vs. LES. $R=D_{i n} / 2$ is the combustion chamber radius. 
network model and appropriate correlations for Nusselt numbers. Radiative heat transfer within the gases and with the wall is neglected. This choice is based on a simplified onedimensional heat transfer model used to estimate the major heat transfer mechanisms taking place at these conditions. For the reacting case simulated in this paper, the total power input is equal to $19.8 \mathrm{~kW}$. Of this power input, about $18 \%$ was estimated to be lost by forced heat convection to the wall and $4 \%$ by radiation, by the end of the quartz tube. More details about this estimate can be found in the Appendix. The truncated domain's outlet boundary condition for pressure uses OpenFOAM's non-reflective condition called wave transmissive $\mathrm{BC}$ inspired by the work of Poinsot and Lelef. 52

\section{VALIDATION}

\section{A. Importance of inflow conditions}

One of the main focus areas of this paper is to explore unstable phenomena occurring in the combustor, and hence it is important to understand the sensitivity of the results to inflow conditions. In the experiment, a flow straightener was added upstream of the swirler. We compared the mean and rms data obtained from PIV for cases with and without the flow straightener under non-reactive conditions. A small difference, less than $7 \%$ on average, in flow statistics was observed. Moreover, a slight change, less than $3 \%$, in the dominant peak in the power spectra, which will be shown later, corresponds to a large scale precessing dynamics, falling below the experimental uncertainty. These results reduce the need for more detailed prescription of the turbulent inlet velocity boundary condition for the case of interest in this paper.

\section{B. Validation: Flow field}

Comparisons between the experimental and LES results are shown for the non-reacting (Figs. 4 and 5) and reacting cases (Figs. 6 and 7). Velocity profiles of four different locations shown in Fig. 8 are used for the comparisons $(\mathrm{x} / \mathrm{R}=0,0.5,1,1.5)$ with $\mathrm{R}=\mathrm{D}_{\text {in }} / 2$ being the combustion chamber radius. The overall agreement is reasonable, but few discrepancies exist. The velocity fluctuations are well captured in the cold flow, but more deviation arises in the reacting case. The average location of the stagnation point shows some discrepancy. For the non-reacting flow, the experiment shows the stagnation point to be located right at the expansion plane, whereas the simulations predict the average stagnation point location to be upstream, inside the inlet tube.

Figure 8 shows the predicted axial velocity distribution in the non-reacting and reacting cases; they qualitatively
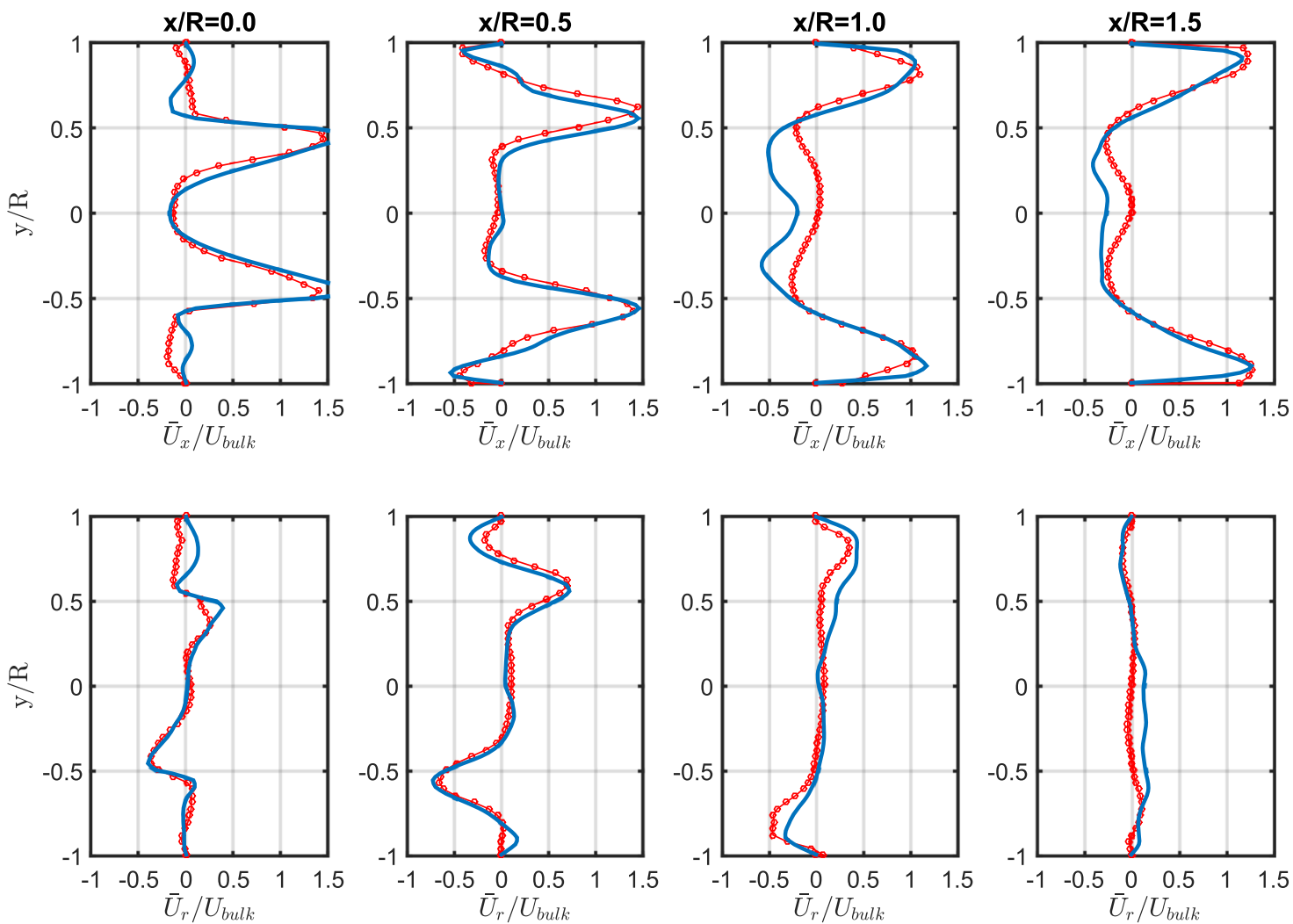

FIG. 6. Reacting mean velocity profiles: Experimental (red circle) vs. LES. $R=D_{i n} / 2$ is the combustion chamber radius. 

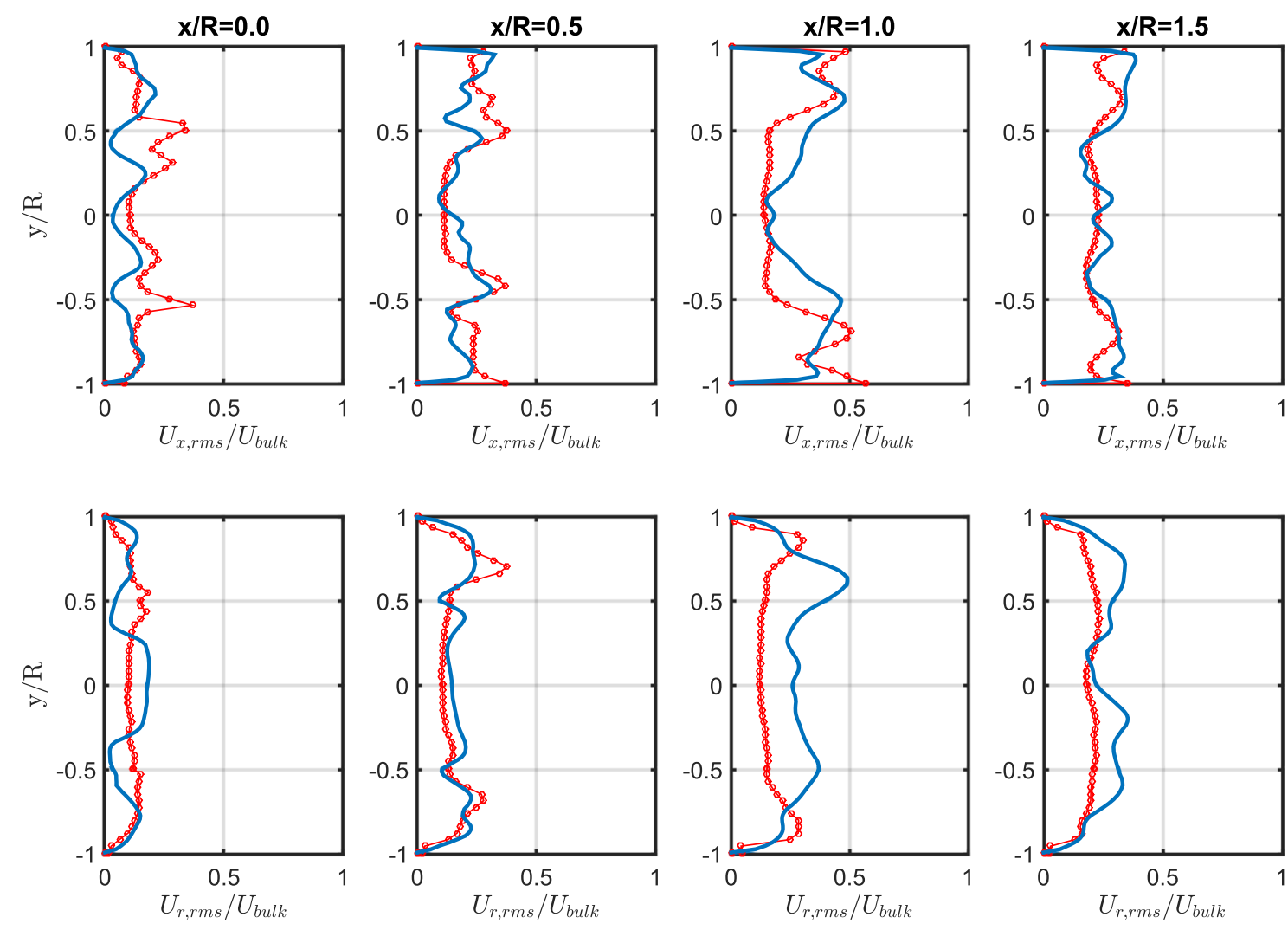

FIG. 7. Reacting RMS velocity profiles: Experimental (red circle) vs. LES. $R=D_{\text {in }} / 2$ is the combustion chamber radius.

capture important swirling flow features. Vortex breakdown in the non-reacting swirling flow (Fig. 8-top) leads to an open recirculation zone, suggesting a cone-type structure, ${ }^{53,54}$ in which the negative axial velocity region extends downstream within the field of view. This compares well with the

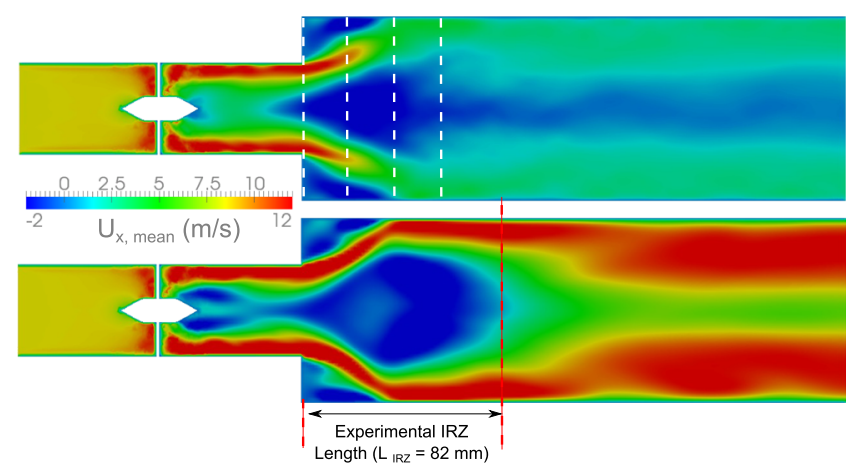

FIG. 8. Axial velocity contours of the non-reacting (top) and reacting (bottom) LES cases. The dashed lines in the top part represent the location of the velocity profiles used for LES validation. The dashed line in the bottom part locates the experimental position of the downstream stagnation point, i.e., the length of the vortex breakdown bubble. experimental PIV data (shown later in the experimental results). The non-reacting case shows also the presence of a comparatively small recirculation zone in the wake of the centerbody (this cannot be confirmed using experimental data as the optical access is restricted to be only downstream of the expansion plane). For the reacting case, vortex breakdown features a closed "bubble" recirculation zone, and the extent of the experimental and numerical recirculation zones compares fairly well. The inner recirculation zone length is measured by the location of the downstream stagnation point, i.e., the axial extend of the zero velocity contour. Additionally, simulations show that the recirculation zone bubble merges with the centerbody's smaller recirculation zone. Moreover, the flame anchors at the center-body which was visually confirmed in the experiment. The merger of the two recirculation zones creates one tubular structure along which the flame can be stabilized.

\section{Validation: ORZ temperature}

Further validation is performed by comparing temperature measurements with predictions for the reacting case $(\phi=0.60)$. The time averaged temperatures in the ORZ $\left(\mathrm{TC}_{1}\right)$ as well as downstream $\left(\mathrm{TC}_{2}\right)$ were probed at the locations shown in Fig. 9(c). The temperature downstream was 

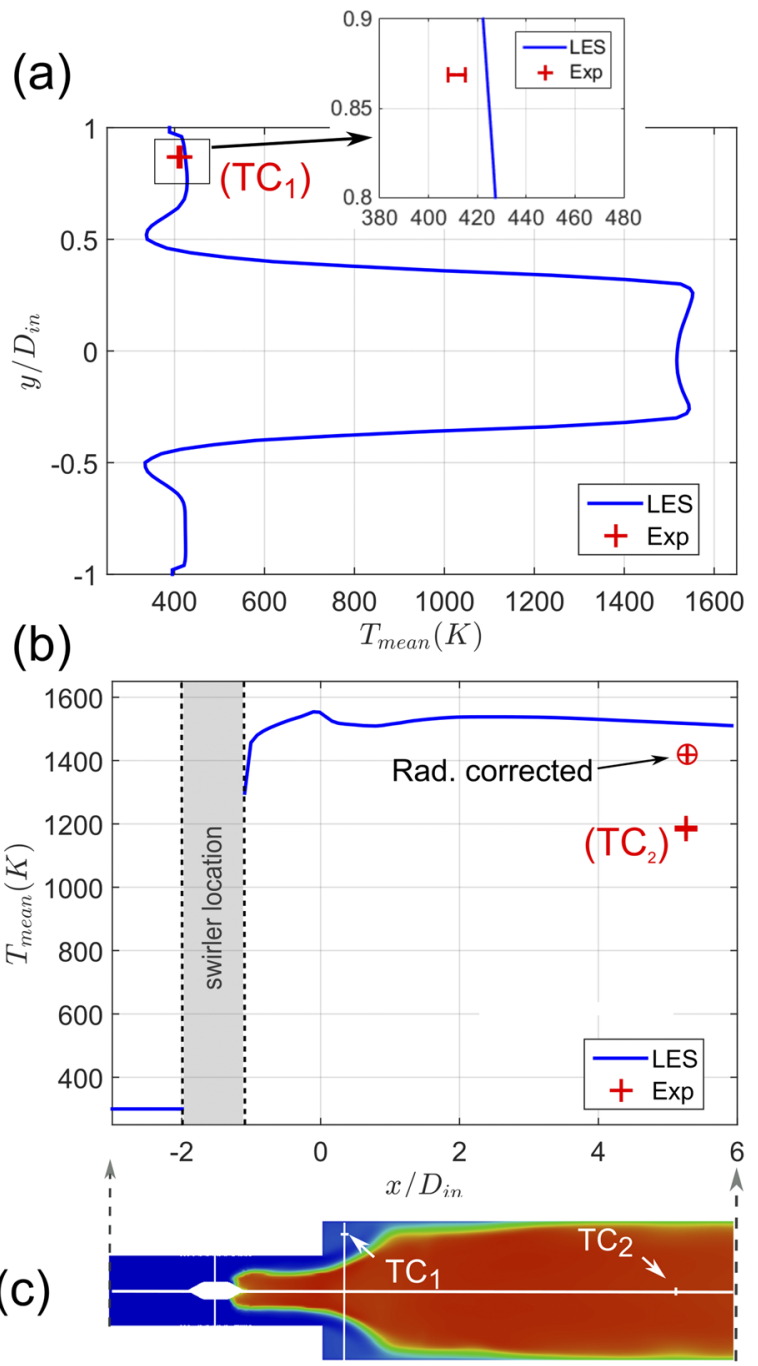

FIG. 9. Comparison of experimental and LES temperature in: (a) the $O R Z\left(T_{1}\right)$ and (b) along the combustor's centerline $\left(\mathrm{TC}_{2}\right)$. The location of $\mathrm{TC}_{1}$ and $\mathrm{TC}_{2}$ is shown in (c). The expansion plane corresponds to $x / D_{\text {in }}=0$. The LES mean temperature contours are shown in (c) with the extracted temperature profiles' location.

corrected for radiation (see the Appendix). The comparisons are shown in Fig. 9(a) for the ORZ temperature and in Fig. 9(c) for the downstream temperature.

While the inlet temperature is ambient and no combustion occurs in the ORZ at this equivalence ratio, the measured value ORZ is $410 \pm 5 \mathrm{~K}$ and the predicted value is $\mathrm{T}_{\text {ORZ,LES }}=423 \mathrm{~K}$ [see Fig. 9(a)]. The temperature rise within the ORZ is explained as follows: The reactant jet travels between the cool IRZ and the burning ORZ, impinges on the side wall, and splits into two parts with one recirculating back into the ORZ. The reactant jet is thus preheated between the sudden expansion and the impingement point. The residence time along this incoming jet (governed by both Re and S) controls the level of preheating. As radiation was not taken into account in the LES model and the computed temperature shows a good agreement with the experimental data, convective heating can be considered the primary mechanism responsible for the ORZ heating.

The downstream temperature measurement $\left(\mathrm{TC}_{2}\right)$ gives an average temperature $\mathrm{T}_{\text {probe }}=1185 \mathrm{~K}$, while the gas surrounding the probe is estimated to be at $\mathrm{T}_{\text {gas }}=1420 \mathrm{~K}$ (correction method in the Appendix). It is important here to make the distinction between $\mathrm{T}_{\text {probe }}$ and $\mathrm{T}_{\text {gas }}$ as the probe is cooled by the colder surroundings through radiative heat loss. The predicted temperature by LES at the same location is $1520 \mathrm{~K}$. Besides the combustion model, the difference from the measured value is potentially due to neglecting heat losses by gaseous radiation; although estimated to be at $2 \%$ of the total power, this can still have a measurable impact on the downstream temperature. For comparison, the adiabatic flame temperature is $\mathrm{T}_{a d, \phi=0.6}=1660 \mathrm{~K}$. The above validation of the downstream temperature has to be taken with care as it suffers from the measurement difficulty and the need to estimate the gas temperature from the measured probe temperature by correcting for the radiative cooling of the probe itself.

\section{Validation: Conclusions}

The comparison of the flow field low order statistics as well as the ORZ temperature shows a fairly good and acceptable agreement although some discrepancies are noted. The unsteady nature of these flows calls for further comparisons, especially the time dependent features. This will be performed throughout the rest of the paper even though the purpose is not purely for validation but rather the extraction of information from simulations that cannot be retrieved experimentally using two-dimensional optical diagnostics.

\section{RESULTS AND ANALYSIS}

In this section, we start by presenting experimental results to unveil the dominant flame and flow dynamics in non-reacting and reacting flow cases. Before doing that, we briefly describe other flame shapes or macrostructures, obtained at different equivalence ratios (at the same inflow conditions) to highlight the range of flame shapes that can arise.

\section{A. Flame macrostructures}

Different flame stabilization modes, often referred to as flame shapes, configurations or macrostructures, are observed when the equivalence ratio is raised starting from the lean blowout limit. These have been documented in previous work using $\mathrm{CH}_{4}-\mathrm{H}_{2}$ blends. ${ }^{43,55}$ Figure 10 displays long exposure (1/4 s exposure time) IR-filtered chemiluminescence images of these macrostructures along with the mean flow field obtained by PIV. At lean conditions, a columnar lifted flame is observed (flame I). This flame extends along the entire combustion chamber (which limits its practicality). The flow is qualitatively similar to that observed in the non-reacting 

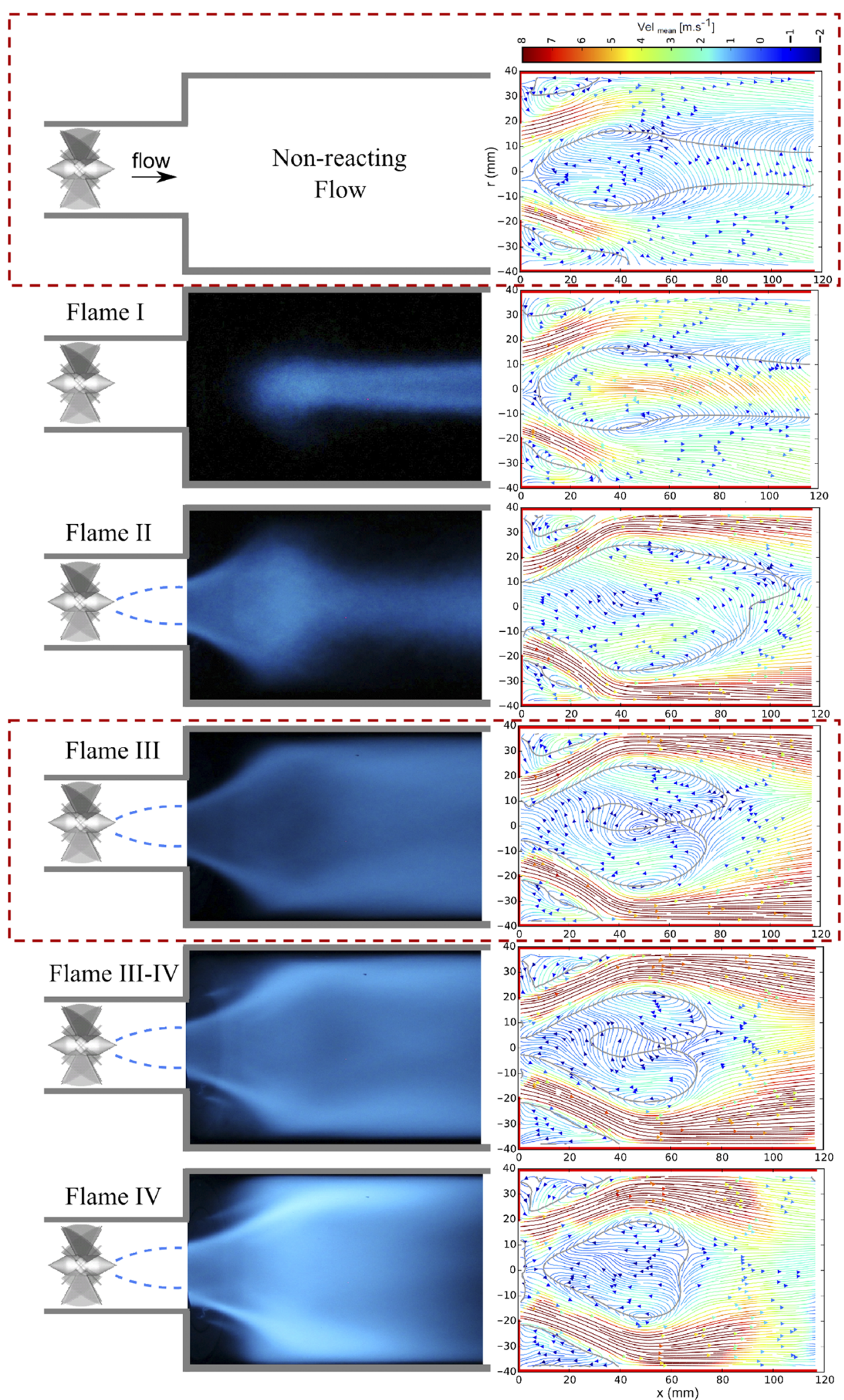

FIG. 10. Experimental long exposure flame chemiluminescence (left column) and mean velocity streamlines (right) for different equivalence ratios at the reference flow conditions $(R e=20000$, $\left.\alpha_{s w}=45^{\circ}, T_{\text {in }}=300 \mathrm{~K}\right)$. The nonreacting and reacting (flame III, $\phi=0.60$ ) cases of interest in this paper are shown in the dashed boxes. Other experimental cases are shown here for reference. Streamlines are colored by the axialradial velocity magnitude multiplied by the sign of the axial velocity. 
case. Both show an open inner recirculation zone characteristic of cone-type vortex breakdown as shown by the zero mean velocity contour. As the equivalence ratio is raised, the flame is visually observed to extend inside the burner tube (not part of the optically accessible region) and anchors at the swirler centerbody (flame II); the mean zero-velocity streamline closes downstream exhibiting a downstream stagnation point and showing a bubble-type recirculation zone. At higher $\phi$, the flame stabilizes along the ISL with a conical shape (flame III). This is the flame we are investigating in this paper. As $\phi$ is raised further, combustion starts taking place first intermittently then continuously in the ORZ and along the OSL (flame IV).

\section{B. Evidence of large scale precessing dynamics and its suppression with combustion}

\section{Pressure-based method for frequency separation}

The dynamic pressure is recorded at the location shown on the experimental setup schematic in Fig. 1, i.e., between the swirler and the expansion plane. This is performed in order to differentiate between acoustic and aerodynamic fluctuations. The recorded signal includes the acoustic and aerodynamic pressure fluctuations. From previous investigations of the same combustor, ${ }^{56}$ the fundamental longitudinal acoustic mode is expected to be around $120 \mathrm{~Hz}$. The power spectral density (PSD) amplitude of the pressure signal for the nonreacting flow is shown in Fig. 11. The PSD amplitude at the reference inflow conditions $(\operatorname{Re}=20000)$ and the reference geometry is shown inside the dotted box of the left column of Fig. 11. At these reference conditions, the PSD shows a relatively large frequency band centered around $120 \mathrm{~Hz}$, which is the expected fundamental frequency of the reference length combustor. Using a single pressure transducer does not differentiate between acoustic and aerodynamic fluctuations. For this reason, we also recorded the pressure signal for different inflow conditions as well as for a different chamber length.

We increase the combustor's length downstream to four times the reference length (long combustor in the right column of Fig. 11) and the Reynolds number (different rows in Fig. 11), to change the acoustic frequencies and flow driven frequencies independently. When changing Re (plots on the left column of Fig. 11), an additional peak appears around $80 \mathrm{~Hz}$ at $\mathrm{Re}=15000$, while the $120 \mathrm{~Hz}$ peak remains unchanged, confirming its acoustic origin (the mean flow has a negligible impact on the acoustic frequency). On the other hand, increasing the length of the chamber, the acoustic frequency is modified, while the frequency peak originating from the flow is unchanged. This method allows the extraction of aerodynamic-based frequencies for different Reynolds numbers even when these overlap with the natural acoustic pressure modes of the combustion chamber, which is the case here. At the reference conditions, a frequency $\mathrm{f}_{\text {flow }}=106 \mathrm{~Hz}$ is extracted.

In Fig. 12, this aerodynamic frequency $\left(\mathrm{f}_{\text {flow }}\right)$ is plotted against the Reynolds number, showing a linear dependency. This leads to a constant Strouhal number defined using the inlet (pre-swirler) bulk velocity and the inlet diameter (St $=\frac{f D_{\text {in }}}{U_{\text {bulk }}} \approx 0.49$ ). Using the same method, the frequency is
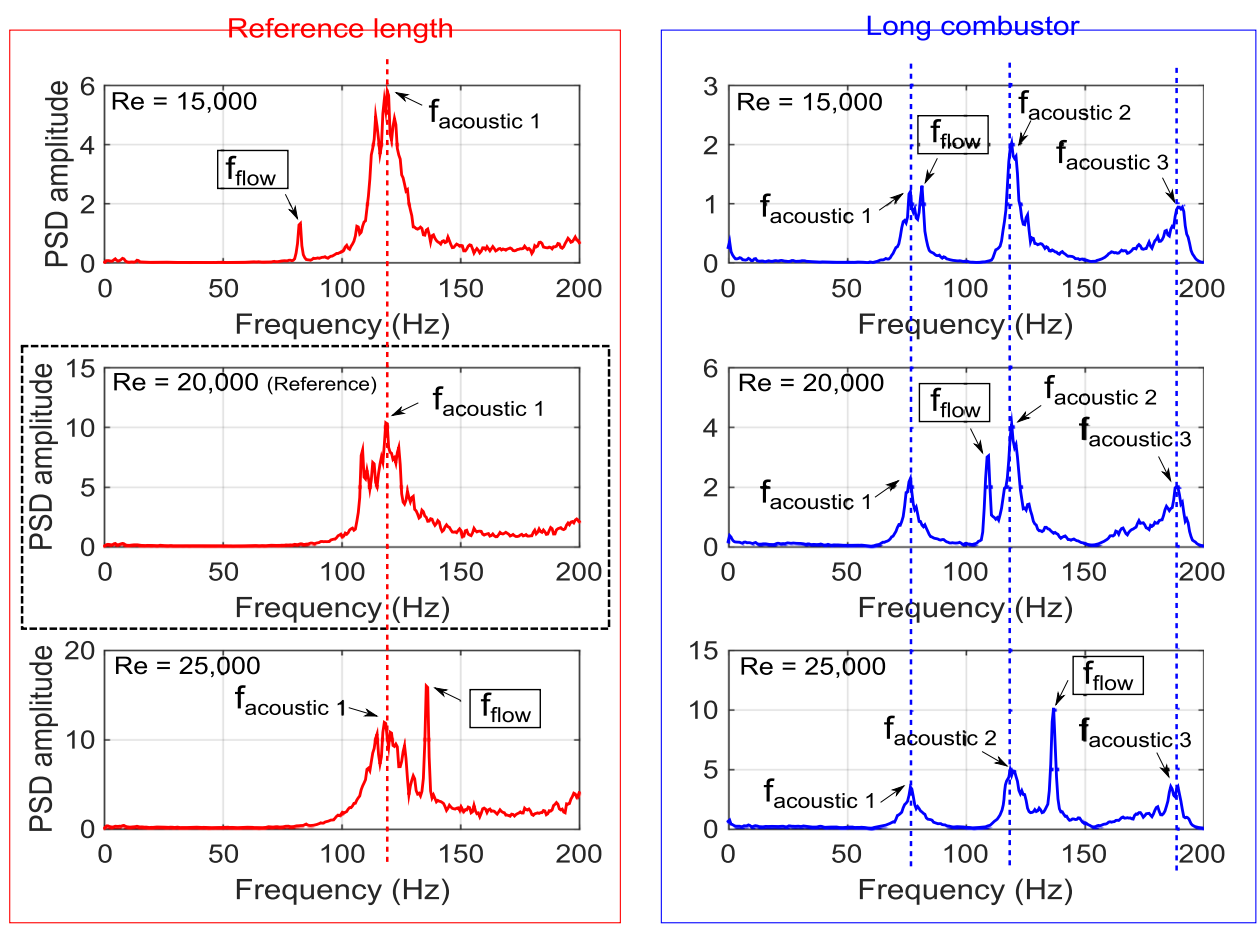

FIG. 11. Power Spectral Density (PSD) of pressure recorded at the dump plane for non-reacting flows at different Reynolds numbers: reference length (left column) and longer chamber (right column). The reference inflow conditions are shown in the dotted box $(\mathrm{Re}=20000$ and reference length). 


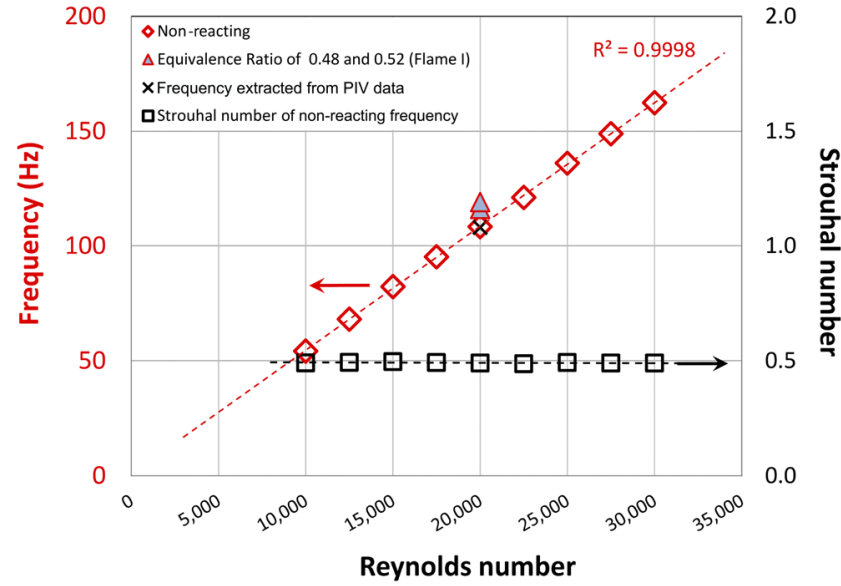

FIG. 12. Pressure-extracted flow frequency and Strouhal number at different Re for non-reacting flow and reacting flow at low equivalence ratio $(0.48$ and 0.52 shown by triangles). For comparison, the PIV-extracted frequency for the non-reacting flow at $R e=20000$ is shown.

extracted for the reacting flows: for flame I, at $\phi=0.48$ and $\phi=0.52$, the frequency is plotted on the same graph (the lean blowoff limit being at $\phi=0.47$ ). The dominant peak appears at a slightly higher frequency for $\phi=0.48$ and $\phi=0.52$ : a $7 \%$ and $10 \%$ increase, respectively, compared to the non-reacting flow. However, pressure measurements at a higher equivalence ratio for flames II, III, and IV (i.e., $\phi$ larger or equal to $0.55)$, including the cases that are the focus of this paper, did not exhibit a tonal spectrum around the frequencies shown above, besides the acoustic frequencies of the chamber, and are therefore not shown here. The coherent fluctuations show the existence of flow driven dynamics under some conditions. However, spatiotemporal information on this motion cannot be extracted from simply measuring the pressure fluctuations.

\section{Flow-based method}

In order to better characterize the above dynamics, the flow data acquired using PIV are analyzed. We find that the instantaneous flow for the non-reacting case is characterized by a large off-axis motion taking place at a precise frequency. In the reacting flow, this large scale motion is only observed for the columnar shaped flame observed from $\phi_{\mathrm{LBO}}$ $=0.47$ to $\phi=0.54$ (flame I). This is consistent with the disappearance of the pressure frequency peak mentioned in Sec. IV B 1 and could indicate suppression of the precessing vortex.

We analyze this motion using the following method: at a distance of $\mathrm{D}_{\text {in }} / 2$ from the expansion plane, the radial location of the axial velocity local maxima $\mathrm{M}_{\text {up }}(\mathrm{t})$ and $\mathrm{M}_{\text {down }}(\mathrm{t})$ are extracted. $\mathrm{M}_{\text {up }}(\mathrm{t})$ and $\mathrm{M}_{\text {down }}(\mathrm{t})$ are shown in Fig. 13 (first row) plotted for the mean axial velocity profile for the non-reacting (left column of Fig. 13) and reacting cases (right column of Fig. 13). These two points are found to oscillate in time at $f_{\text {jet }}=108 \mathrm{~Hz}$ for the non-reacting case which is similar to the
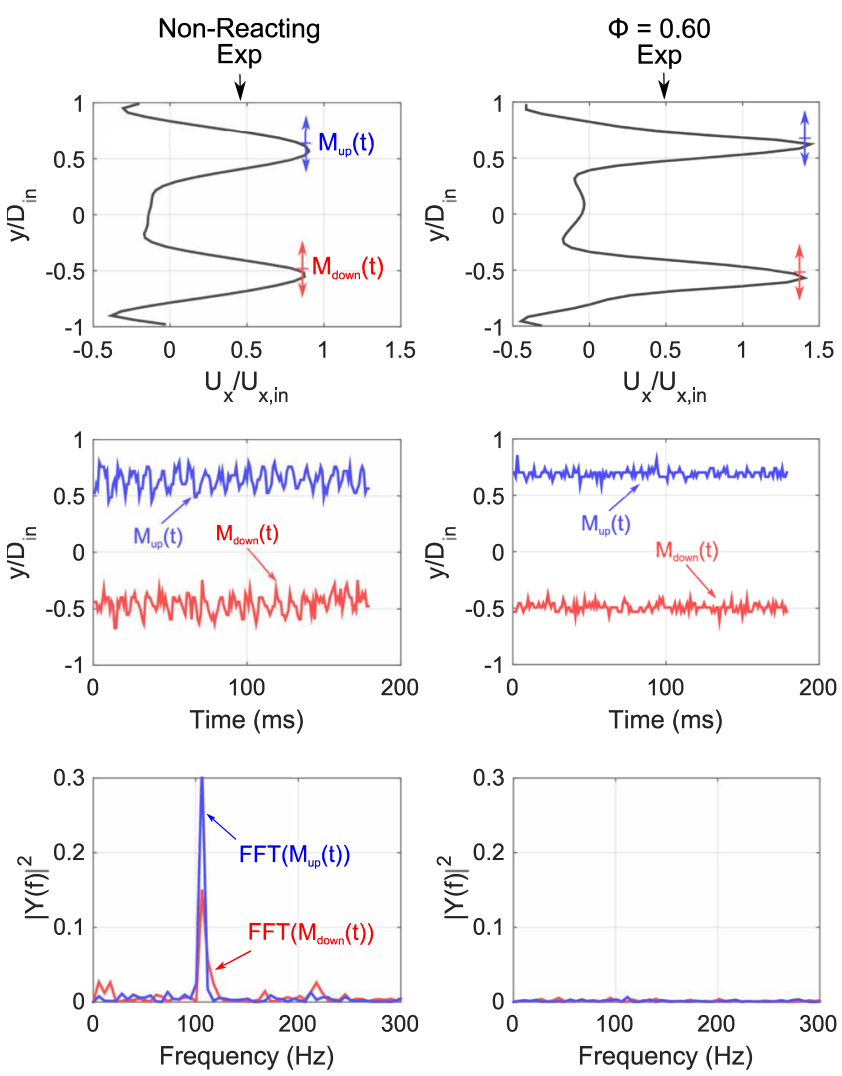

FIG. 13. Dynamics of the incoming jet maxima (at $x=D_{\text {in }} / 2$ ) for the non-reacting (left column) and the reacting case at $\phi=0.60$ (right column) extracted from PIV data. The first row represents the mean velocity profile and location of maxima $\left(M_{u p}\right.$ and $\left.M_{\text {down }}\right)$. The second row shows $M_{u p}(t)$ and $M_{\text {down }}(t)$ signals. The third row shows the Fast Fourier Transform (FFT) of $\mathrm{M}_{\text {up }}(\mathrm{t})$ and $\mathrm{M}_{\text {down }}(\mathrm{t})$.
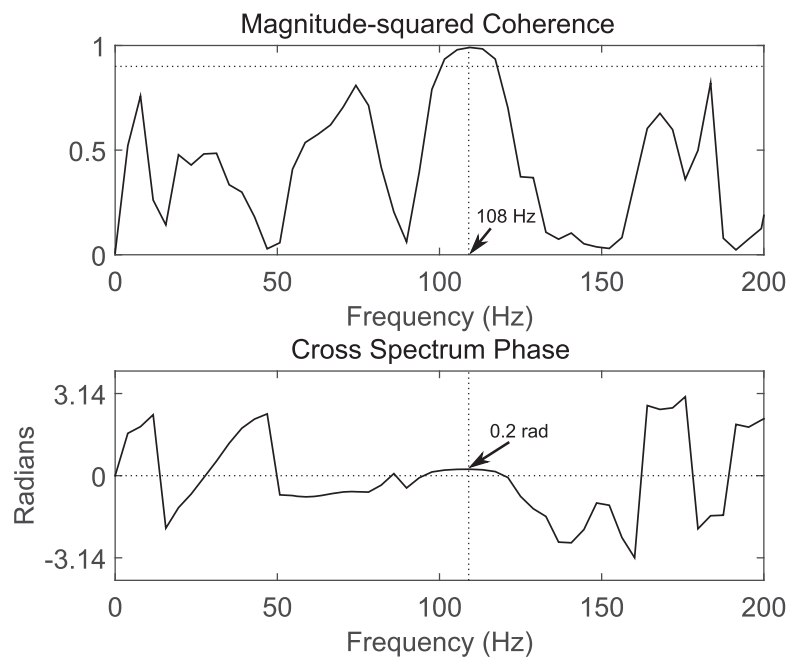

FIG. 14. Phase between $M_{u p}(t)$ and $M_{\text {down }}(t)$ showing in-phase oscillations at $108 \mathrm{~Hz}$. 
frequency extracted using the pressure-based method at the same flow conditions. The phase delay between $\mathrm{M}_{\text {up }}(\mathrm{t})$ and $\mathrm{M}_{\text {down }}(\mathrm{t})$ (Fig. 14) shows that the two jets not only oscillate at the same frequency $f_{j e t}$ but also move in phase, suggesting that the entire flow field is rotating around the combustor's centerline. The similarity between this frequency and the ones extracted above using the pressure-based method shows that the frequencies measured in Fig. 12 are indeed flow driven events and these are due to a large scale offaxis rotation. The frequency of flow oscillation for flame I at $\phi=0.51$ is found at $117 \mathrm{~Hz}$. When increasing the equivalence ratio beyond $\phi=0.54$ (including $\phi=0.6$ which is the focus of this paper), this large scale dynamics disappears as soon as the flame macrostructures change and the flame extends to the swirler centerbody located inside the burner tube. At $\phi=0.60$, there is only a weak and non-coherent motion of $\mathrm{M}_{u p}(\mathrm{t})$ and $\mathrm{M}_{\text {down }}(\mathrm{t})$ as it can be seen in Fig. 13 (right column). Our results are consistent with the hypothesis that the suppression of the precessing vortex core is a consequence of the attachment of the flame to the centerbody which, in turn, creates strong radial density and temperature gradients in the inlet region. ${ }^{31}$

\section{Vortical structures along the shear layers}

The precessing dynamics is only present in the nonreacting flow and very lean lifted flames (flame I). For the conical ISL stabilized flame at $\phi=0.60$, this large scale motion of the entire field disappears; however, other flow and flame structures and dynamics are still observed. These are mainly
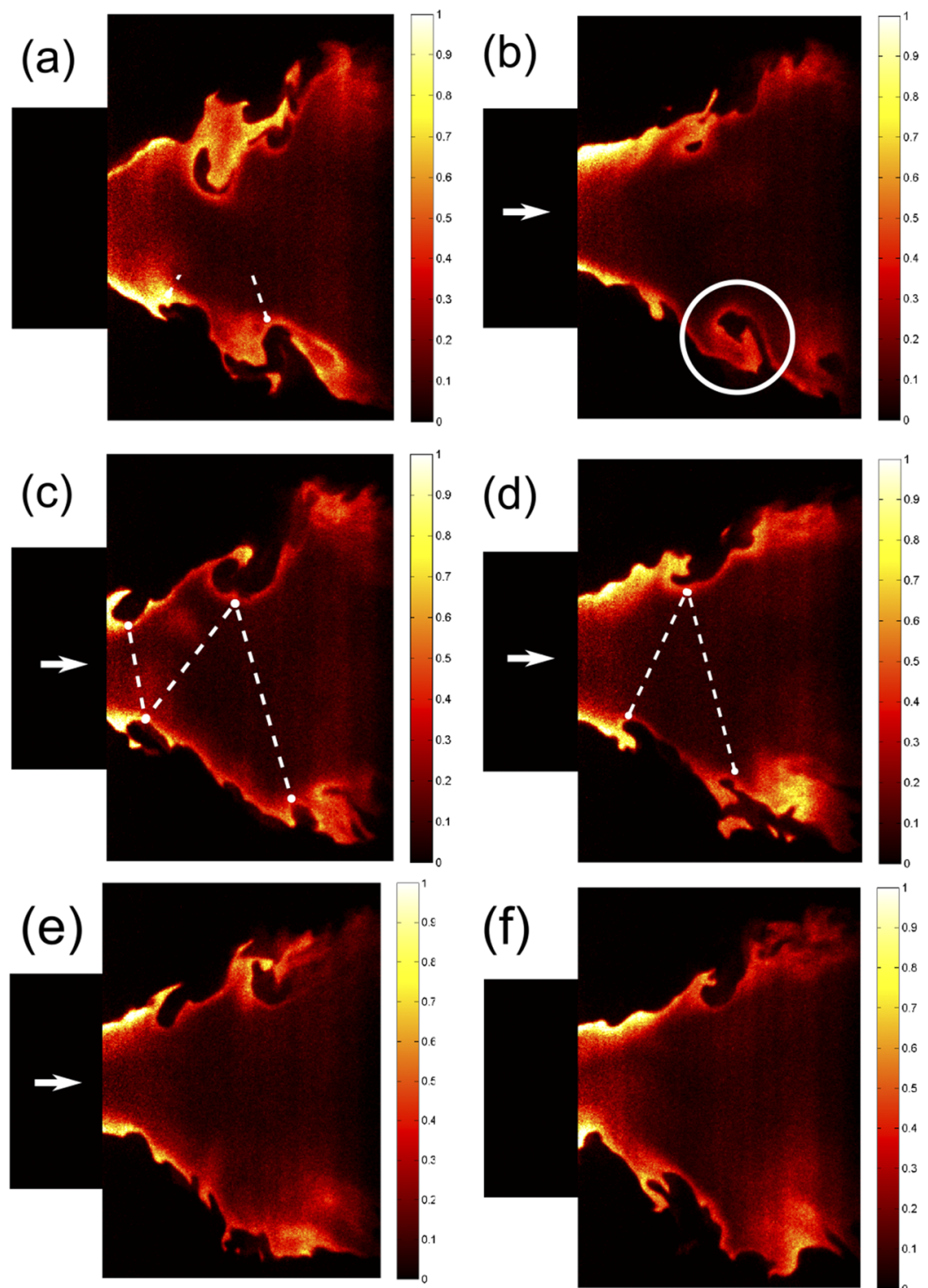
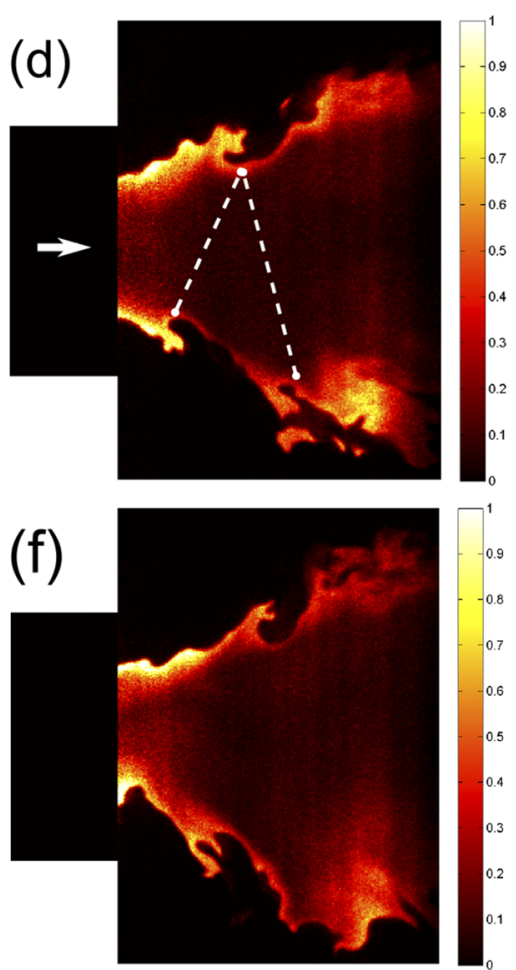

FIG. 15. Non-consecutive OH-PLIF images (a) through (f) of ISL flame for $\mathrm{CH}_{4} /$ air at $\phi=0.60$. 

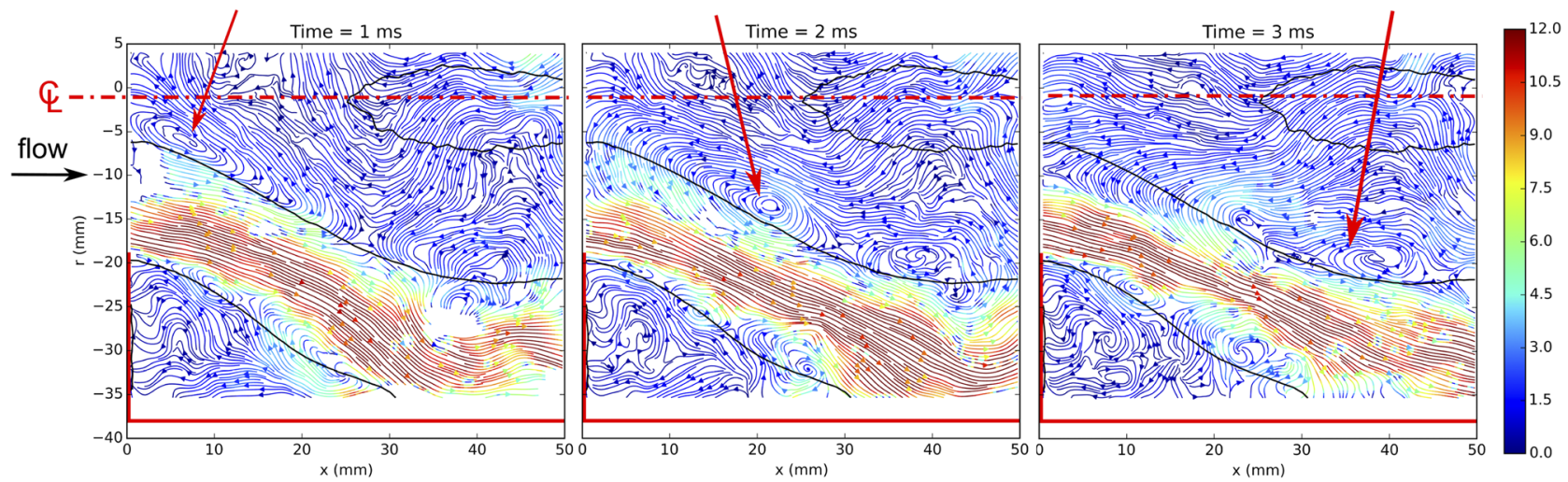

FIG. 16. Consecutive streamline plots colored by $x$-r velocity magnitude (colored by the sign of axial velocity) at $\phi=0.60$. Solid black line corresponds to the mean zero velocity contour. Solid red border corresponds to the chamber wall. Dotted-dashed red line shows the chamber's centerline.
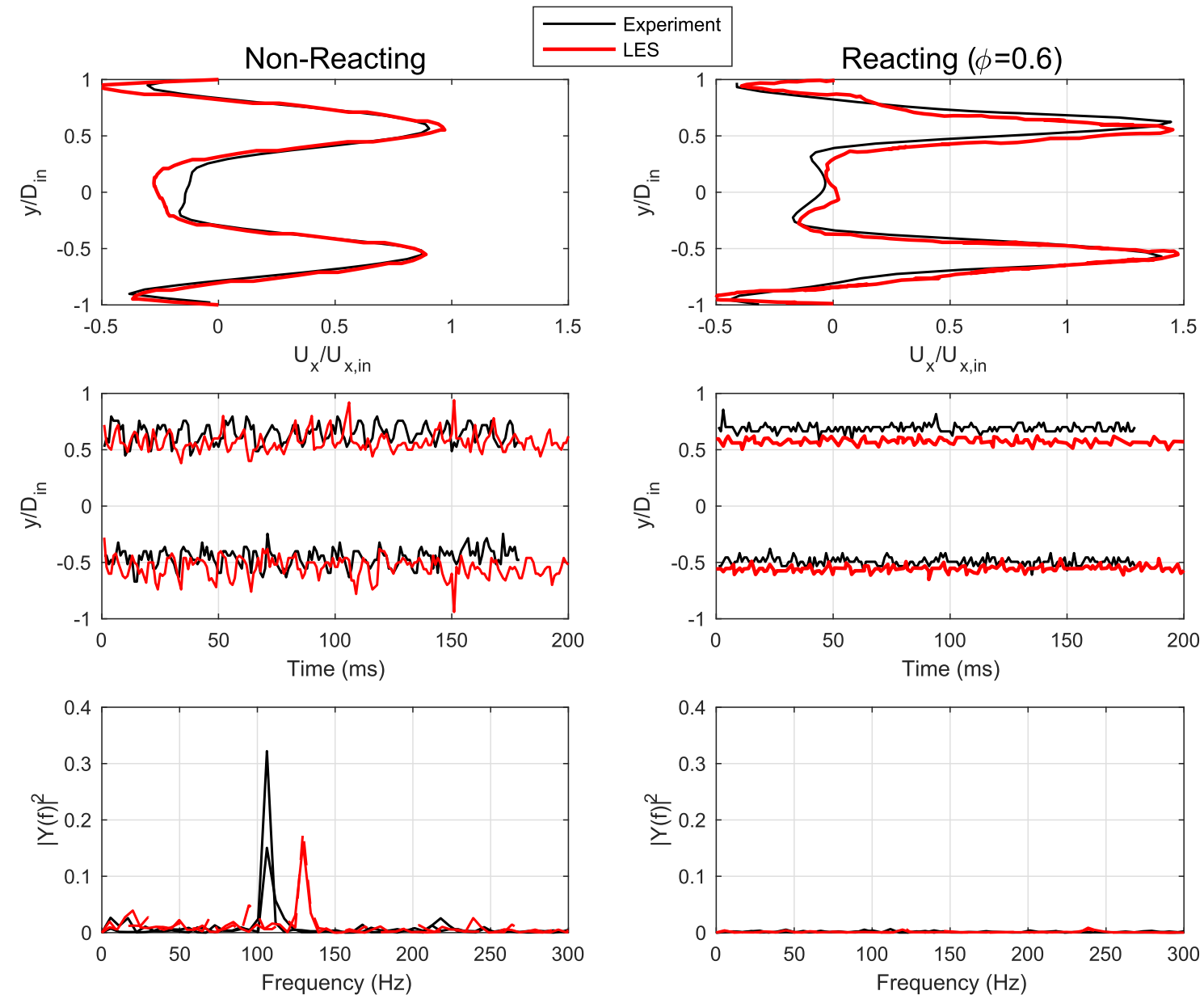

FIG. 17. Dynamics of the incoming jet maxima (at $x=D_{\text {in }} / 2$ ) for non-reacting (left column) and reacting case (right column) extracted from LES and compared to the experimental data. The first row represents the mean velocity profile and location of maxima $\left(M_{u p}\right.$ and $\left.M_{\text {down }}\right)$. The second row shows $M_{\text {up }}(t)$ and $M_{\text {down }}(t)$ signals. The third row shows the Fast Fourier Transform $(F F T)$ of $\mathrm{M}_{u p}(\mathrm{t})$ and $\mathrm{M}_{\text {down }}(\mathrm{t})$. 
vortical structures along the inner shear layer, and in this section, we show experimental evidence for their existence. We will mainly rely on optical diagnostics (PLIF, PIV) as pressure measurements did not allow the identification of any large scale dynamics besides the natural acoustic frequencies of the chamber.

\section{OH-PLIF evidence}

The ISL stabilized flame's micro-structure is now interrogated using OH-PLIF. A set of non-consecutive images from these data is shown in Fig. 15. The images are taken at a $10 \mathrm{~Hz}$ repetition rate which might not be sufficient to resolve flow and flame dynamics but still can reveal some important features. These images confirm the ISL stabilization of the instantaneous flame front. More importantly, we observe the flame to be wrinkled by what appears to be vortices along the ISL as can be clearly seen in Fig. 15(c). In addition, the flame roll-ups are not aligned on the same axial position (see dashed lines in Fig. 15), suggesting the presence of a spiral or helical vortex similar to what was described in the introduction. The flame appears to be entrained by such a large scale structure leading to flame wrinkles of the order of $\delta \approx 10-12 \mathrm{~mm}$, one order of magnitude larger than the laminar flame thickness.

Flame snapshots similar to those shown in Fig. 15 strongly suggest the existence of organized vortical structures. While it seems clear from their shape that these flame roll ups are due to the flow structure in the shear layer, their origin is less straightforward.

\section{PIV evidence}

PIV measurements are performed to confirm the existence of vortical structures along the ISL. In Fig. 16, the instantaneous planar velocity streamlines (smaller field of view focused on half chamber width) at 3 consecutive times are displayed, with $1 \mathrm{~ms}$ separating each snapshot. A vortex can be observed at the first time step and tracked in time along the shear layer. It is likely that these vortices are the ones responsible for large scale wrinkling of the flame observed in Fig. 15.

\section{COMPLEMENTARY LES RESULTS}

The experimental observation allowed us to identify some important qualitative aspects of the precessing dynamics. However, it only suggested the existence of flame roll-ups and vortical structures along the shear layer without full characterization (frequency and shape for example). In this section, we perform complementary LES to gain further insight into their origin and dynamics. The first step is to determine whether the large scale precessing dynamics shown experimentally in the non-reacting flow as well as its absence at larger equivalence ratio for the reactive cases can also be demonstrated in the simulation.

\section{A. Precessing motion and its suppression}

The simulated non-reacting swirling flow case at the reference conditions does exhibit the large scale precessing

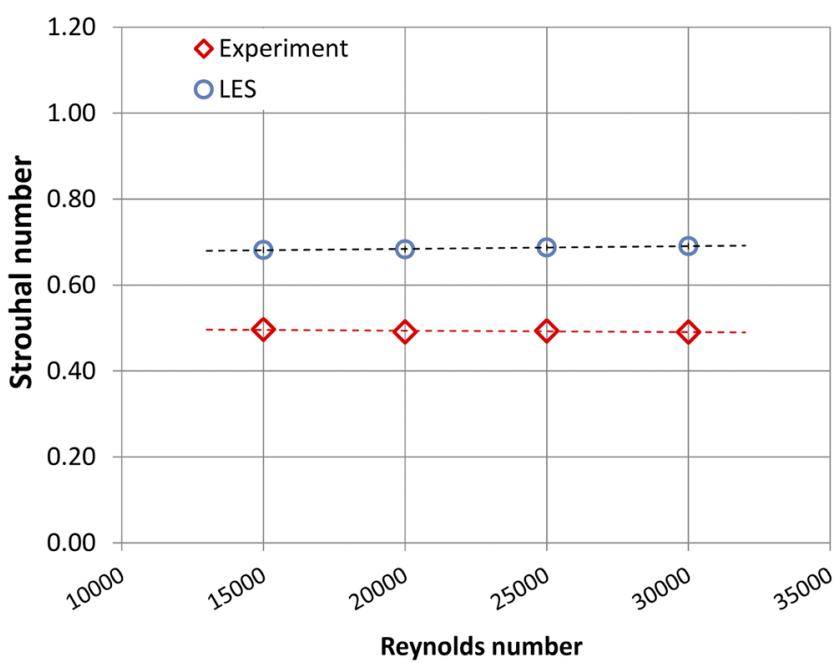

FIG. 18. LES vs. experimental precessing Strouhal for the non-reacting flow case (frequency extracted using the velocity method for LES and the pressure method for the exp. data)

motion highlighted by the experiments. A corresponding plot to Fig. 13, which illustrated the precessing motion from experimental data, is obtained using the simulation results shown

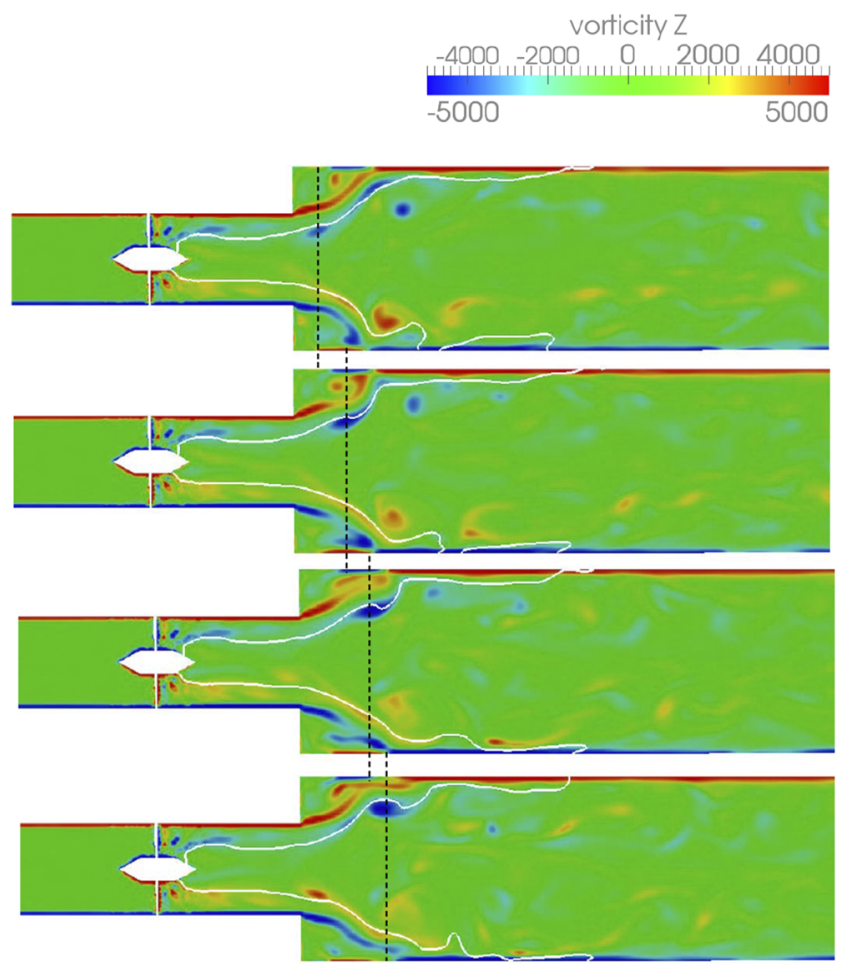

FIG. 19. LES of reacting case $\phi=0.60$ featuring the out of plane vorticity as well as the progress variable contour $c=0.8$ (white line) as a way to locate the flame front. $1 \mathrm{~ms}$ separates consecutive frames. 
in Fig. 17 and compared to the experimental data. The radial locations of the two velocity maxima, which are captured well by the simulation, oscillate in-phase similarly to what was experimentally observed. For both the non-reacting and reacting cases, the oscillation amplitude is in good agreement with the experimental data. For the simulated non-reacting case, the oscillation takes place at a frequency of $129 \mathrm{~Hz}$. This frequency should be compared with the experimental precessing frequency of $108 \mathrm{~Hz}$. In the simulations of the reacting case, the flame stabilizes along the inner shear layer and extends to the swirler centerbody which is visually seen in the experiment as well. Moreover, similar to the experiment, the precessing motion is damped. The fact that these two characteristics are well reproduced by the LES gives us some confidence that the simulations can be predictive in terms of the flame transition between different flame modes presented in Fig. 10.
The precessing motion was previously shown to scale with the Reynolds number and to lead to a constant Strouhal number (Fig. 12). The same is captured by the simulation as shown in Fig. 18. However, as noted above, some discrepancies can be seen in the frequency itself. Simulation results show a constant Strouhal number at $\mathrm{St}_{\mathrm{LES}}=0.59$, higher than the experimental value $\mathrm{St}_{\mathrm{EXP}}=0.49$. In the current analysis, this is considered acceptable since the scaling with the inflow conditions, i.e., different Re's, is well captured.

\section{B. Flame wrinkling and the helical vortex}

The flame wrinkling along the ISL that was observed experimentally is now numerically investigated. Although the three dimensional field is available in simulations, we start by analyzing a $2 \mathrm{D}$ view similar to what was performed in the experiment. Longitudinal cross sections of the domain

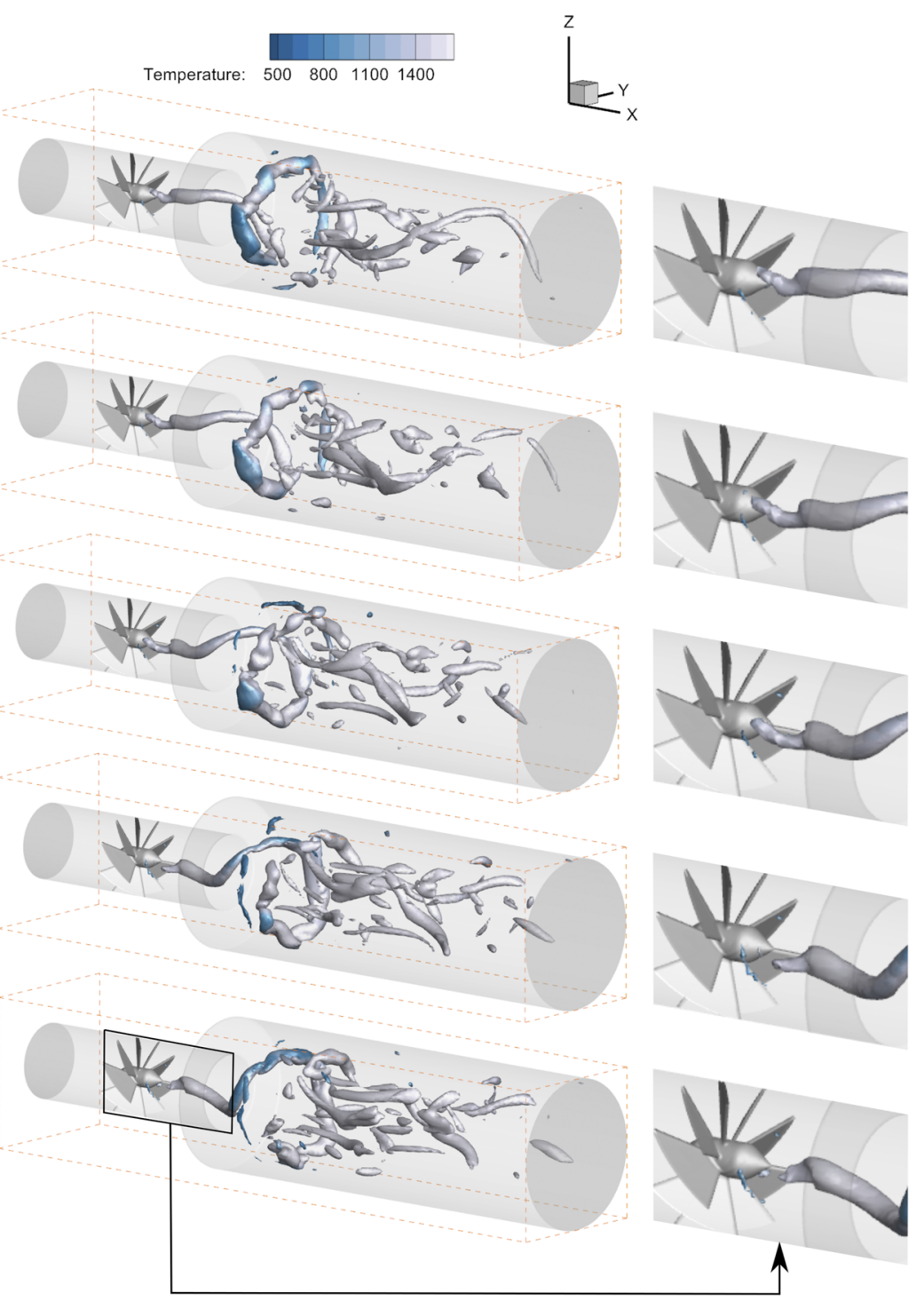

FIG. 20. Three-dimensional vortex core identification using the lambda-2 criterion (here colored by temperature) showing the helical vortex core for the reacting case with a time interval of $1 \mathrm{~ms}$ between each image. 
are considered, and the azimuthal vorticity is plotted along with the flame contour. The result is shown in Fig. 19. In the upper ISL, a clockwise vortex is present (located by the vertical black dashed line). This vortex is close to the flame, entrains it in its motion, and wraps it around. After $1 \mathrm{~ms}$ and $2 \mathrm{~ms}$, a vortex appears slightly downstream with a stronger wrinkling of the flame. These vortices can be tracked in time and in a 2D-domain; a vortex in the same upper ISL would appear again at approximately the same location every 7-8 ms, i.e., with frequency around $133 \mathrm{~Hz}$.

We now extract vortex cores using a lambda-2 detection algorithm. ${ }^{57}$ The results are plotted in Fig. 20. We see that the vortices observed in Fig. 19 are in fact 2D cuts of a continuous helical vortex core (HVC) extending to the swirler centerbody. The HVC is mostly aligned with the combustor centerline as it gets closer to the swirler centerbody. As we move away from the centerbody, the vortex core deviates from the geometrical centerline and starts winding around in a conical helical motion. Vorticity transitions from being predominately streamwise in the inlet tube to predominately tangential after the expansion. The streamwise portion of the vortex inside the inlet tube extends to the swirler centerbody and has a weak off-axis motion. Overall, the HVC dynamics is mainly a cork screw like motion where the axis of the cork screw remains close to the geometrical centerline. This motion means that the convection of vortices downstream, as seen in Fig. 19, is only a plotting artefact due to the 2D cut; a 2D vortex will appear at the same location on the interrogated plane when the helix performs an entire revolution around the combustor's centerline.

A natural question so far is whether such a vortex exists in the non-reacting flow. In the non-reacting flow, a similar HVC can be extracted, but it is governed by more complex dynamics: in addition to the solid body rotation of the HVC observed for the reacting flow, the non-reacting HVC has an off-axis motion of the vortex core around the geometric centerline entraining the entire flow in a precessing motion. The inlet tube portion of the $\mathrm{VC}$ does not remain close to the centerline as in the reacting flow but rotates around it. The frequency of rotation of this vortex is equal to $129 \mathrm{~Hz}$, which is the same frequency extracted from the jet motion in Fig. 17. We also see occasionally a double helix forming, with two helical cores rotating around each other and merging; this specific non-reacting flow dynamics will not be studied here as we are focusing on the reacting case. It will be the subject of future investigations.

Figures 21 and 22 illustrate the important difference between the reacting flow and the non-reacting one: for the non-reacting flow (left column), the vortex core is closer to the wall than to the centerline. The precessing radius is shown in the bottom row of Fig. 22; for the reacting case (right column), the precessing radius is smaller. The streamwise vorticity illustrating the location of the vortex core shows that the latter is strictly inside the flame tube. It is also adjacent to the negative velocity region illustrated in the figure by the zero axial velocity contour; both the negative velocity region and
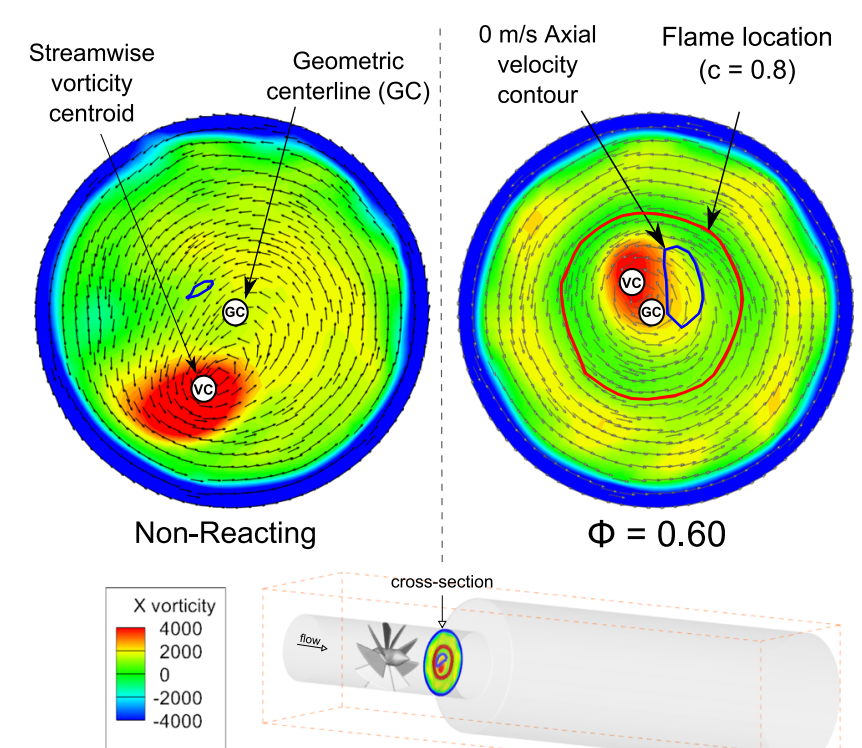

FIG. 21. Superposition of LES streamwise vorticity, velocity field, zero axial velocity contours, and the flame (progress variable $c=0.8$ ) showing the location of the vortex core relatively to the centerline, the negative axial velocity region, and the flame. Slice location: $x=D_{\text {in }}$ from the swirler, inside the inlet tube. Left column: non-reacting flow. Right column: reacting flow.

the vortex core are in a circular motion around the geometric centerline.

The above results show that combustion has an important effect of the helical structures as is also confirmed in the literature. In the cases under investigation, its effect is to damp the precessing motion but maintain the helical vortex structure and its solid body rotation around the centerline. With combustion, the vortex core extends to the swirler centerbody and remains relatively close to the centerline; in other words, from a precessing helical vortex core (P-HVC) in the non-reacting flow, the structure becomes a non-precessing helical vortex core (NP-HVC). The vortex core in the reacting case also appears inside the flame tube away from the shear layer where the flame stabilizes; this can be clearly seen in the instantaneous snapshot in Fig. 23(b). This has the important implication that this flow structure is not caused by shear layer instability but rather a helical instability of the swirling flow (discussed in the introduction of this paper). The NP$\mathrm{HVC}$ is inside the high temperature product zone. The dilatation effect of heat release is a known vorticity sink mechanism and is likely responsible for the low and dampened rotation radius of the vortex core around the geometric centerline (precessing). The baroclinic term (not quantified here) could also be part of the reason as we expect the density gradient to be radial and the pressure gradient to be mainly axial.

Although inside the flame manifold in the inlet tube, the vortex core impacts the flame more downstream: as we move downstream of the centerbody, the core moves away from 


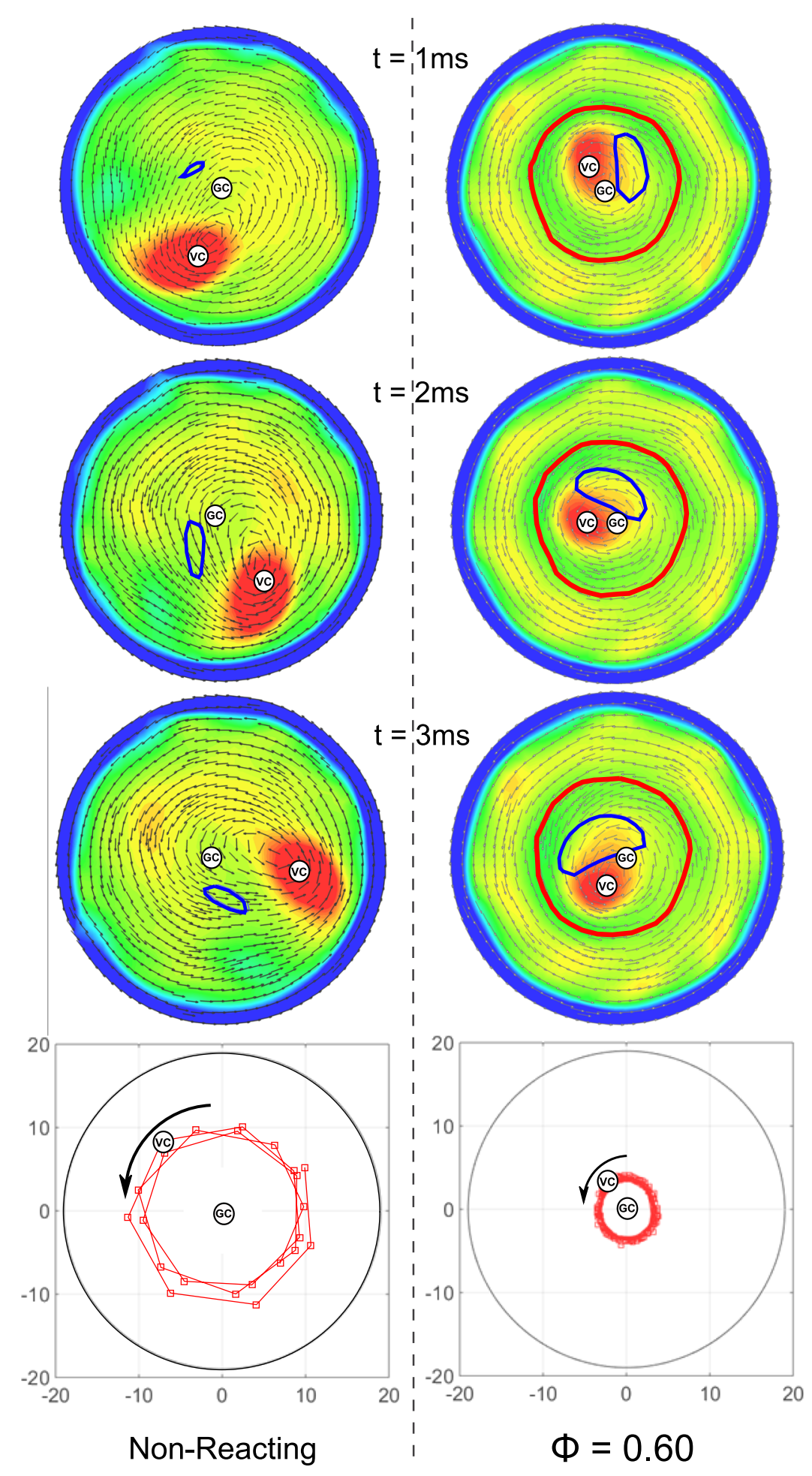

FIG. 22. Superposition of LES streamwise vorticity, velocity field, zero axial velocity contours, and the flame (progress variable $c=0.8$ ) showing the location of the vortex core relatively to the centerline, the negative axial velocity region, and the flame. Slice location: $x=D_{\text {in }}$ from the swirler, inside the inlet tube. Left column: non-reacting flow. Right column: reacting flow. Time between snapshots: $1 \mathrm{~ms}$. Bottom row: trajectory of the vortex core over time.

the centerline and acquires its cone-spiral shape. The core gets closer to the vortex breakdown's inner shear layer where the flame is stabilized and starts interacting with the flame, ultimately wrapping it in a helical roll-up. This phenomenon can be observed in Fig. 24 where the flame contour is shown along with the position of the HVC. This explains the large 

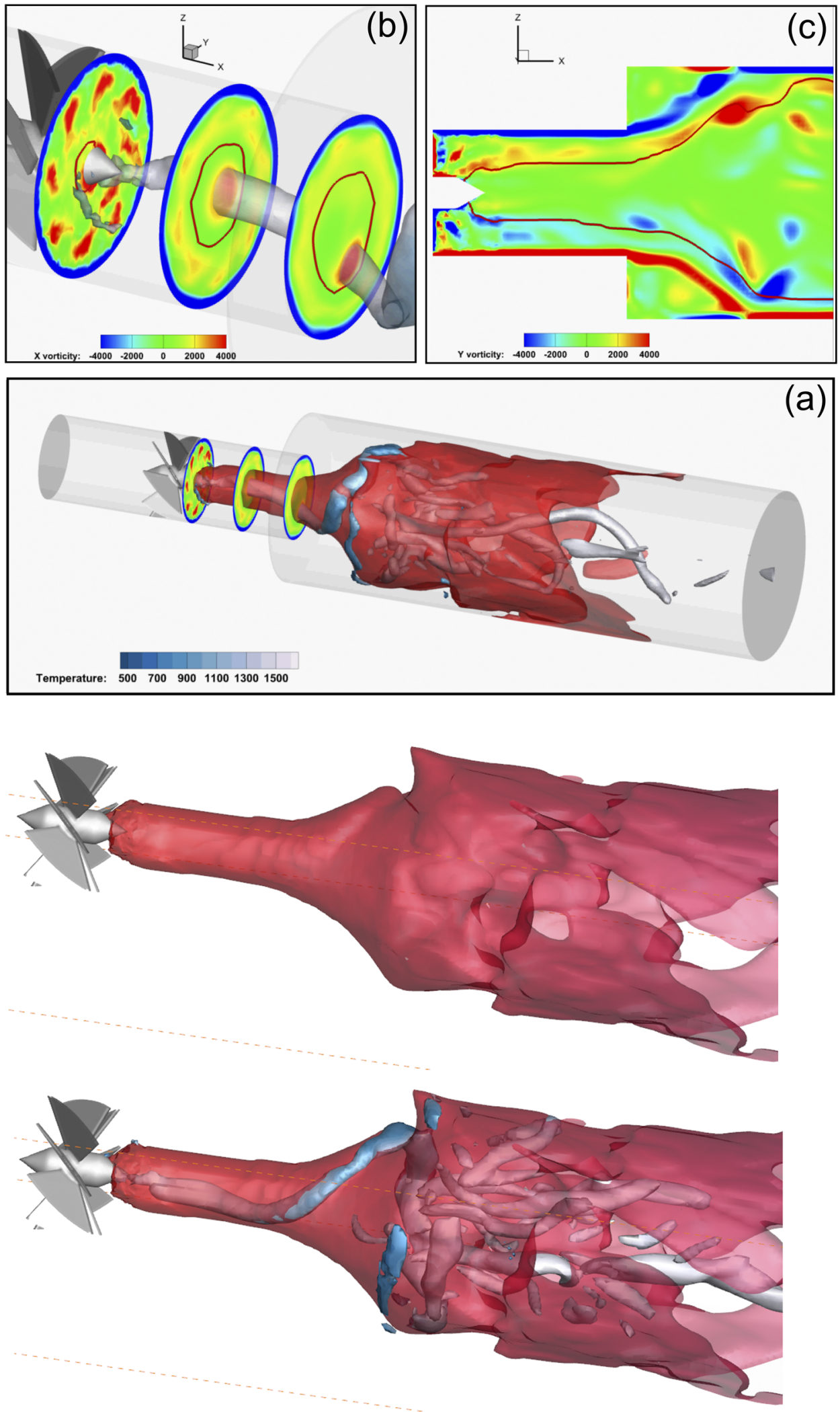

FIG. 23. (a) Instantaneous flame contour (progress variable $c=0.8$ ) for the reacting case $(\phi=0.60)$ with helical vortex core identified using the lambda-2 criterion. (b) Zoom on the region between the swirler and the sudden expansion showing the vortex core originating from the swirler centerbody and remaining aligned with the geometrical centerline and inside the flame tube. Cross sections show axial vorticity contours. (c) Azimuthal vorticity contours and instantaneous flame contour (progress variable $c=0.8$ ) showing the flame roll-up by the helical vortex core when it reaches the inner shear layer.
FIG. 24. Instantaneous flame contour for the reacting case $(\phi=0.60)$ : (top) without helical vortex core showing a helical roll-up of the flame, and (bottom) with helical vortex core showing the location of the vortex with respect to the flame. Helical vortex core identified using the lambda-2 criterion. 
scale and organized wrinkles observed experimentally using OH-PLIF (Fig. 15).

\section{CONCLUDING REMARKS}

In this study, we investigated important swirling flow and flame dynamics and its impact on the premixed flame. We relied in our analysis on a combination of 2D steady state experimental (PIV, OH-PLIF) and complementary 3D unsteady numerical (LES) tools to unveil complex dynamics. In the process of this investigation, the LES code was first validated against flow field and temperature measurements and areas of potential future improvements were indentified. A reduced chemical mechanism was used in this study ${ }^{49}$ containing 7 species and 5 reactions. The mechanism although rather simplified in order to exactly reproduce some special flow characteristics such as the downstream temperature can give an acceptable approximation of the adiabatic flame temperature and the laminar burning velocity in a laminar premixed same setup. A more detailed mechanism could improve the results; however, it would not be practical for the simulation of large scale applications intended for industrial purposes that this study targets.

We reached the following conclusions through our study:

1. The precessing dynamics are present for the nonreacting flow and lifted columnar flames observed at low equivalence ratios; it is largely damped for the ISL stabilized flame. Both experimental results and LES capture this behavior. The suppression of the off-axis motion is concomitant with the change in vortex breakdown type and also the flame attachment to the swirler centerbody.

2. A helical vortex core exists in both non-reacting and reacting cases; it originates at the swirler centerbody. In the non-reacting case, its off-axis precessing motion dominates its dynamics. In the reacting flow, the vortex core rotates in place, i.e., its dynamics is dominated by a "cork screw" solid body type rotation with only a weak off-axis motion around the combustor's centerline. The effect of combustion is to damp the precessing motion, but the HVC structural integrity and its coherent rotation are maintained.

3. LES results show that the "cork screw" shape and motion of the HVC are responsible for the large scale flame wrinkling along the inner shear layer and explain the OH-PLIF experimental observations.

In future work, we will be focusing on fine-tuning the LES model to improve its prediction of the different frequencies highlighted in this study. In addition, outer shear layer instabilities are likely present in this reacting flow but at a smaller scale and strength compared to the helical vortex core highlighted above. Finer LES grid/filter simulations will be used to extract OSL structures and investigate their interaction with the helical vortex studied in this paper. Finally, the occasional appearance of a double-helix, only observed in the non-reacting flow and briefly mentioned in this paper, will be further investigated.

\section{ACKNOWLEDGMENTS}

The authors would like to thank the King Fahd University of Petroleum and Minerals in Dhahran, Saudi Arabia, for funding the research reported in this article through the Center for Clean Water and Clean Energy at MIT and KFUPM under Project No. R12-CE-10. K.V. would like to acknowledge the support of the Engineering and Physical Science Research Council through the grant EP/S001824/1.

\section{APPENDIX: TEMPERATURE CORRECTION AND 1D HEAT TRANSFER MODELING}

The temperature is measured for the reference reacting case $\left(\mathrm{CH}_{4}\right.$ at $\left.\phi=0.6\right)$ using a K-type sheathed thermocouple $\mathrm{TC}_{2}$ (sheath diameter is $1 / 16$ in., a response time around $1 \mathrm{~s}$ and sampling frequency of $20 \mathrm{~Hz}$ ) inserted from the tube end along the centerline reaching a distance $\mathrm{x}=0.2 \mathrm{~m}$ from the expansion plane (middle of the quartz tube). The measurement is repeated twice, and steady state values are recorded and averaged in time to obtain $\mathrm{T}_{\text {probe }}$. During this measurement, the probe loses heat by radiation to the relatively colder chamber wall and a radiation correction is required. A simplified one dimensional steady state thermal resistance network model is used to estimate heat losses and the temperature at the wall by considering a thermal energy balance for the wall: internal forced convection to the wall, radiation from the products to the wall, conduction through the quartz tube thickness, radiation out to the environment, and finally natural convection cooling of the tube. The wall resistance is neglected compared to other resistances, and the wall temperature is assumed uniform across the wall. Heat is lost by natural convection and radiation exchange with the surroundings at ambient temperature. Using this model, for each gas temperature $\left(\mathrm{T}_{\text {gas }}\right)$, a wall temperature $\left(\mathrm{T}_{\text {wall }}\right)$ can be solved for. Such a model allows also to estimate the relative weight of each heat transfer mode.

The downstream temperature measurement ( $\left.\mathrm{T}_{\text {probe }}\right)$ repeated twice gives $1181 \mathrm{~K}$ and $1188 \mathrm{~K}$. Knowing $\mathrm{T}_{\text {probe }}$ and by using a heat balance on the probe, which exchanges heat with the products flowing around it at $\mathrm{T}_{\text {gas }}$ but also looses heat to the wall at $\mathrm{T}_{\text {wall }}$ by radiation, $\mathrm{T}_{\text {gas }}$ and the associated $\mathrm{T}_{\text {wall }}$ are estimated. The corrected gas temperature is thus $\mathrm{T}_{\text {gas }}=1420 \mathrm{~K}$. This estimate of the gas temperature is used for LES result validations. Figure 25(a) shows the estimated wall and probe temperatures as a function of the gas temperature using the 1D model. In Fig. 25(b), the contribution of each mode of heat transfer from the hot gases is estimated. The total power input is equal to $19.8 \mathrm{~kW}$ (for $\mathrm{CH}_{4}$ air at $\phi=0.60$ and $\mathrm{Re}=20$ 000). About $18 \%$ is estimated to be lost by forced heat convection to the wall and $4 \%$ by radiation which led us to neglect radiation modeling in LES calculations. 

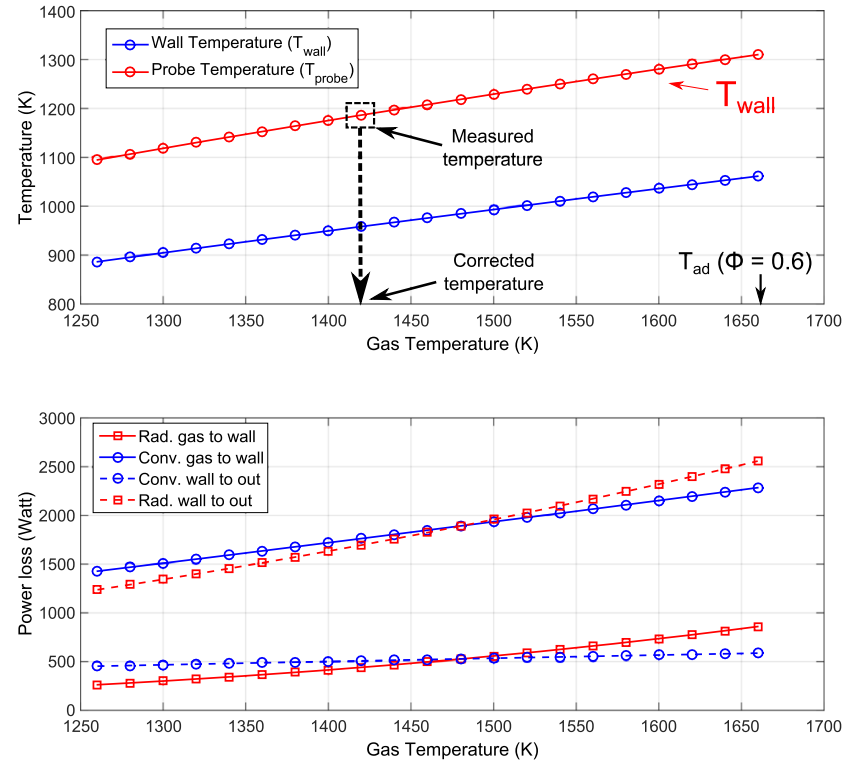

FIG. 25. Top: Gas and wall temperatures obtained from the radiation loss correction of the temperature measured by the thermocouple. Bottom: Modeled losses mechanism for half the combustor length (where the temperature measurement is performed). The black arrows correspond to the estimates based on the measured temperature.

\section{REFERENCES}

${ }^{1}$ R. Rotunno, "The fluid dynamics of tornadoes," Annu. Rev. Fluid Mech. 45, 59 (2013).

${ }^{2}$ H. Ha, W. Choi, H. Park, and S. J. Lee, "Effect of swirling blood flow on vortex formation at post-stenosis," Proc. Inst. Mech. Eng., Part H 229, 175183 (2015).

${ }^{3}$ R. T. Whitcomb, "A design approach and selected wind tunnel results at high subsonic speeds for wing-tip mounted winglets," NASA Technical Report NASA-TN-D-8260 (1976).

${ }^{4}$ B. A. Dobbs, D. B. Wigmore, and R. E. Niggemann, "Swirl flow heat exchanger with reverse spiral configuration," U.S. patent 4,883,117 (November 28, 1989).

${ }^{5} \mathrm{~W}$. Barth, "Design and layout of the cyclone separator on the basis of new investigations," Brenn. Warme Kraft 8, 1-9 (1956).

${ }^{6} \mathrm{C}$. M. Coats, "Coherent structures in combustion," Prog. Energy Combust. Sci. 22, 427-509 (1996).

${ }^{7}$ S. J. Shanbhogue, S. Husain, and T. Lieuwen, "Lean blowoff of bluff body stabilized flames: Scaling and dynamics," Prog. Energy Combust. Sci. 35, 98120 (2009).

${ }^{8}$ S. M. Correa, "Power generation and aeropropulsion gas turbines: From combustion science to combustion technology," Symp. (Int.) Combust. 27, 1793-1807 (1998).

${ }^{9}$ A. K. Gupta, D. G. Lilley, and N. Syred, "Swirl flows," in Tunbridge Wells (Abacus Press, Kent, England, 1984), Vol. 1, p. 488.

${ }^{10} \mathrm{E}$. Tangermann, M. Pfitzner, M. Konle, and T. Sattelmayer, "Large-eddy simulation and experimental observation of combustion-induced vortex breakdown," Combust. Sci. Technol. 182, 505-516 (2010).

${ }^{11}$ S. Terhaar, K. Oberleithner, and C. O. Paschereit, "Key parameters governing the precessing vortex core in reacting flows: An experimental and analytical study," Proc. Combust. Inst. 35, 3347-3354 (2015).

${ }^{12}$ M. G. Hall, "Vortex breakdown," Annu. Rev. Fluid Mech, 4, 195-218 (1972).
${ }^{13} \mathrm{~N}$. Syred and J. M. Beer, "Combustion in swirling flows: A review," Combust. Flame 23, 143-201 (1974).

${ }^{14}$ D. G. Lilley, "Swirl flows in combustion: A review," AIAA J. 15, 1063-1078 (1977).

${ }^{15}$ S. Leibovich, "The structure of vortex breakdown," Annu. Rev. Fluid Mech. 10, 221-246 (1978).

${ }^{16}$ M. Escudier, "Vortex breakdown: Observations and explanations," Prog. Aerosp. Sci. 25, 189-229 (1988).

17J. M. Delery, "Aspects of vortex breakdown," Prog. Aerosp. Sci. 30, 1-59 (1994).

${ }^{18} \mathrm{O}$. Lucca-Negro and T. O’Doherty, "Vortex breakdown: A review," Prog. Energy Combust. Sci. 27, 431-481 (2001).

${ }^{19} \mathrm{~N}$. Syred, "A review of oscillation mechanisms and the role of the precessing vortex core (PVC) in swirl combustion systems," Prog. Energy Combust. Sci. 32, 93-161 (2006).

${ }^{20} \mathrm{Y}$. Huang and V. Yang, "Dynamics and stability of lean-premixed swirl-stabilized combustion," Prog. Energy Combust. Sci. 35, 293-364 (2009).

${ }^{21}$ S. Candel, D. Durox, T. Schuller, J.-F. Bourgouin, and J. P. Moeck, "Dynamics of swirling flames," Annu. Rev. Fluid Mech. 46, 147-173 (2014).

${ }^{22}$ C. Oliver Paschereit, E. Gutmark, and W. Weisenstein, "Coherent structures in swirling flows and their role in acoustic combustion control," Phys. Fluids 11, 2667-2678 (1999).

${ }^{23}$ H. K. Moffatt, "Spiral structures in turbulent flow," in New Approaches and Concepts in Turbulence (Springer, 1993), pp. 121-129.

${ }^{24} \mathrm{~F}$. Gallaire and J.-M. Chomaz, "Instability mechanisms in swirling flows," Phys. Fluids 15, 2622-2639 (2003).

${ }^{25}$ C. E. Cala, E. C. Fernandes, M. V. Heitor, and S. I. Shtork, "Coherent structures in unsteady swirling jet flow," Exp. Fluids 40, 267-276 (2006).

${ }^{26} \mathrm{Y}$. Huang and V. Yang, "Effect of swirl on combustion dynamics in a leanpremixed swirl-stabilized combustor," Proc. Combust. Inst. 30, 1775-1782 (2005).

${ }^{27}$ X. Lu, S. Wang, H.-G. Sung, S.-Y. Hsieh, and V. Yang, "Large-eddy simulations of turbulent swirling flows injected into a dump chamber," J. Fluid Mech. 527, 171-195 (2005).

${ }^{28}$ K. Oberleithner, M. Sieber, C. N. Nayeri, C. O. Paschereit, C. Petz, H.-C. Hege, B. R. Noack, and I. Wygnanski, "Three-dimensional coherent structures in a swirling jet undergoing vortex breakdown: Stability analysis and empirical mode construction," J. Fluid Mech. 679, 383-414 (2011).

${ }^{29}$ A. M. Steinberg, C. M. Arndt, and W. Meier, "Parametric study of vortex structures and their dynamics in swirl-stabilized combustion," Proc. Combust. Inst. 34, 3117-3125 (2013).

${ }^{30} \mathrm{~K}$. Oberleithner, S. Terhaar, L. Rukes, and C. Oliver Paschereit, "Why nonuniform density suppresses the precessing vortex core," J. Eng. Gas Turbines Power 135, 121506 (2013).

${ }^{31}$ K. Oberleithner, M. Stöhr, S. H. Im, C. M. Arndt, and A. M. Steinberg, "Formation and flame-induced suppression of the precessing vortex core in a swirl combustor: Experiments and linear stability analysis," Combust. Flame 162, 3100 (2015).

${ }^{32}$ B. Coriton and J. H. Frank, "Experimental study of vorticity-strain rate interaction in turbulent partially premixed jet flames using tomographic particle image velocimetry," Phys. Fluids 28, 025109 (2016).

${ }^{33}$ R. Jarpala, Naga Venkata Sai Aditya Burle, M. Voleti, and R. Sadanandan, "Effect of swirl on the flame dynamics and pollutant emissions in an ultralean non-premixed model gas turbine burner," Combust. Sci. Technol. 189, 1832-1848 (2017)

${ }^{34}$ C. D. Slabaugh, C. N. Dennis, I. Boxx, W. Meier, and R. P. Lucht, " 5 kHz thermometry in a swirl-stabilized gas turbine model combustor using chirped probe pulse femtosecond cars. Part 2. Analysis of swirl flame dynamics," Combust. Flame 173, 454-467 (2016).

${ }^{35}$ Q. An, W. Y. Kwong, B. D. Geraedts, and A. M. Steinberg, "Coupled dynamics of lift-off and precessing vortex core formation in swirl flames," Combust. Flame 168, 228-239 (2016).

${ }^{36}$ C. M. Arndt, M. Stöhr, M. J. Severin, C. Dem, and W. Meier, "Influence of air staging on the dynamics of a precessing vortex core in a dual swirl 
gas turbine model combustor," in 53rd AIAA/SAE/ASEE Joint Propulsion Conference (AIAA, 2017), p. 4683.

${ }^{37}$ P. S. Volpiani, T. Schmitt, and D. Veynante, "Large eddy simulation of a turbulent swirling premixed flame coupling the TFLES model with a dynamic wrinkling formulation," Combust. Flame 180, 124-135 (2017).

${ }^{38}$ T. Jaravel, E. Riber, B. Cuenot, and G. Bulat, "Large eddy simulation of an industrial gas turbine combustor using reduced chemistry with accurate pollutant prediction," Proc. Combust. Inst. 36, 3817-3825 (2017).

${ }^{39}$ D. Moëll, D. Lörstad, and X.-S. Bai, "LES of hydrogen enriched methane/air combustion in the SGT-800 burner at real engine conditions," in ASME Turbo Expo 2018: Turbomachinery Technical Conference and Exposition (American Society of Mechanical Engineers, 2018), p. V04BT04A023.

${ }^{40} \mathrm{~S}$. Li, Y. Zheng, M. Zhu, D. M. Martinez, and X. Jiang, "Large-eddy simulation of flow and combustion dynamics in a lean partially premixed swirling combustor," J. Energy Inst. 90, 120-131 (2017).

${ }^{41}$ A. N. Lipatnikov, V. A. Sabelnikov, S. Nishiki, and T. Hasegawa, "Combustion-induced local shear layers within premixed flamelets in weakly turbulent flows," Phys. Fluids 30, 085101 (2018).

${ }^{42}$ A. N. Lipatnikov, V. A. Sabelnikov, S. Nishiki, and T. Hasegawa, "Does flame-generated vorticity increase turbulent burning velocity?," Phys. Fluids 30, 081702 (2018).

${ }^{43}$ S. Taamallah, S. J. Shanbhogue, and A. F. Ghoniem, "Turbulent flame stabilization modes in premixed swirl combustion: Physical mechanism and Karlovitz number-based criterion," Combust. Flame 166, 19-33 (2016).

${ }^{44}$ S. Taamallah, N. W. Chakroun, H. Watanabe, S. J. Shanbhogue, and A. F. Ghoniem, "On the characteristic flow and flame times for scaling oxy and air flame stabilization modes in premixed swirl combustion," Proc. Combust. Inst. 36, 3799-3807 (2017).

45J. M. Beer and N. A. Chigier, Combustion Aerodynamics (Krieger, Malabar, FL, 1983).

${ }^{46}$ G. Kewlani, Z. Labry, N. Abani, S. Shanbhogue, and A. Ghoniem, "Large eddy simulations and experimental investigation of flow in a swirl stabilized combustor," in 50th AIAA Aerospace Sciences Meeting Including the New Horizons Forum and Aerospace Exposition (American Institute of Aeronautics and Astronautics, 2012), p. 178.
${ }^{47}$ G. Kewlani, K. Vogiatzaki, S. Shanbhogue, and A. F. Ghoniem, "Validation study of large-eddy simulations of wake stabilized reacting flows using artificial flame thickening approaches," in 51st AIAA Aerospace Sciences Meeting Including the New Horizons Forum and Aerospace Exposition (American Institute of Aeronautics and Astronautics, 2013), p. 169.

${ }^{48}$ G. Kewlani, S. Shanbhogue, and A. Ghoniem, "Investigations into the impact of the equivalence ratio on turbulent premixed combustion using particle image velocimetry and large eddy simulation techniques: 'v' and 'm' flame configurations in a swirl combustor," Energy Fuels 30, 3451 (2016).

${ }^{49} \mathrm{~W}$. P. Jones and R. P. Lindstedt, "Global reaction schemes for hydrocarbon combustion," Combust. Flame 73, 233-249 (1988).

${ }^{50}$ O. Colin, F. Ducros, D. Veynante, and T. Poinsot, "A thickened flame model for large eddy simulations of turbulent premixed combustion," Phys. Fluids 12, 1843 (2000).

${ }^{51} \mathrm{~J} .-\mathrm{P}$. Legier, T. Poinsot, and D. Veynante, "Dynamically thickened flame LES model for premixed and non-premixed turbulent combustion," in Proceedings of the Summer Program (Center for Turbulence Research, 2000), pp. 157-168.

${ }^{52} \mathrm{~T}$. J. Poinsot and S. K. Lelef, "Boundary conditions for direct simulations of compressible viscous flows," J. Comput. Phys. 101, 104-129 (1992).

${ }^{53}$ T. Sarpkaya, "Turbulent vortex breakdown," Phys. Fluids 7, 2301-2303 (1995).

${ }^{54}$ P. Billant, J. Chomaz, and P. Huerre, "Experimental study of vortex breakdown in swirling jets," J. Fluid Mech. 376, 183-219 (1998).

${ }^{55} \mathrm{~S}$. Taamallah, Z. A. LaBry, S. J. Shanbhogue, and A. F. Ghoniem, "Thermoacoustic instabilities in lean premixed swirl-stabilized combustion and their link to acoustically coupled and decoupled flame macrostructures," Proc. Combust. Inst. 35, 3273 (2015)

${ }^{56}$ S. Taamallah, Z. A. LaBry, S. J. Shanbhogue, M. A. M. Habib, and A. F. Ghoniem, "Correspondence between 'stable' flame macrostructure and thermo-acoustic instability in premixed swirl-stabilized turbulent combustion," J. Eng. Gas Turbines Power 137, 071505 (2015).

${ }^{57} \mathrm{~J}$. Jeong and F. Hussain, "On the identification of a vortex," J. Fluid Mech. 285, 69-94 (1995). 